\title{
Multiple access interference in MIMO-CDMA systems under Rayleigh fading: statistical characterization and applications
}

\author{
Khalid Mahmood', Syed Muhammad Asad' ${ }^{1}$, Muhammad Moinuddin², \\ Azzedine Zerguine ${ }^{3 *}$ and Lahouari Cheded ${ }^{4}$
}

\begin{abstract}
A major limiting factor in the performance of multiple-input-multiple-output (MIMO) code division multiple access (CDMA) systems is multiple access interference (MAl) which can reduce the system's capacity and increase its bit error rate (BER). Thus, a statistical characterization of the MAl is vital in analyzing the performance of such systems. Since the statistical analysis of MAI in MIMO-CDMA systems is quite involved, especially when these systems are fading, existing works in the literature, such as successive interference cancellation (SIC) or parallel interference cancellation (PIC), employ suboptimal approaches to detect the subscriber without involving the need for MAI statistics. The knowledge of both MAI and noise statistics plays a vital role in various applications such as the design of an optimum receiver based on maximum likelihood (ML) detection, evaluation of the probability of bit error, calculation of the system's capacity, evaluation of the outage probability, estimation of the channel's impulse response using methods including the minimum mean-square-error (MMSE), the maximum likelihood(ML), and the maximum a posteriori probability (MAP) criterion. To the best of our knowledge, there is no existing work that explicitly evaluates the statistics of the MAI-plus-noise in MIMO fading channels. This constitutes the prime objective of our proposed study here. In this work, we derive the expressions for the probability density function (PDF) of MAI and MAl-plus-noise in MIMO-CDMA systems in the presence of both Rayleigh fading channels and additive white Gaussian noise. Moreover, we evaluate the probability of the bit error rate in the presence of optimum reception using a ML receiver. Our theoretical findings can provide a reliable basis for both system design and various performance analyses of such systems. Our simulation results show that the theoretical findings are very well substantiated.
\end{abstract}

\section{Introduction}

In spite of their numerous advantages, MIMO-CDMA systems suffer from a major drawback in MAI which can reduce their capacity and increase their BER, thus resulting in a degraded system performance. Hence, a statistical analysis of MAI becomes a very important factor in the performance analysis of these systems.

In CDMA systems, each user is assigned a unique spreading code. These orthogonal codes should ideally provide perfect isolation from other subscribers so as to maintain error-free communication among all users. However, in reality, the orthogonality between these codes

\footnotetext{
*Correspondence: azzedine@kfupm.edu.sa

3 Department of Electrical Engineering, King Fahd University of Petroleum and Minerals, Dhahran 31261, Kingdom of Saudi Arabia
}

Full list of author information is available at the end of the article is difficult to preserve due to asynchronism and channel delay spread at the receiving end. While both asynchronism and channel delay spread exist on the uplink, only channel delay spread can be seen on the downlink of the channel. A correlation receiver, which in a multipath channel turns into a Rake receiver, cannot perfectly separate the signals in the case of multiple users. This lack of separation leads to MAI causing a system performance degradation, which may lead to unacceptable error performances for moderate user loads.

Most of the reported research work is done on characterization of single-input single-output (SISO) CDMA systems and is based only on approximations, such as the standard Gaussian approximation (SGA) [1], improved Gaussian approximation (IGA) [2] and simplified IGA (SIGA) [3]. The central limit theorem is applied in SGA 
to get an approximate sum of additive white Gaussian noise processes (AWGNP). These approximations are widely used because of their ease of application but can exhibit major drawbacks. For example, the SGA overestimates the system's performance, thus making the use of approximations an even more severe problem when the number of users is small [2]. The Standard Hermite polynomial error correction method was employed in [4] to improve the accuracy of SGA. In [5], the improved Gaussian approximation (IGA) method was used based on both the derivation of the conditional characteristic function of MAI and on bounds on its error probability for binary direct-sequence spread-spectrum multiple access (DS-SSMA) systems. In the case where the number of users is low, IGA has outperformed the SGA [2] but at the cost of an increased in computational complexity which makes it a major limitation for this method. The IGA was later simplified and renamed as the simplified IGA (SIGA) in [6].

Another approach adopted by researchers to find the BER of the DS/SSMA scheme is to ignore MAI completely. Most of these techniques are basically an extension of previously studied inter-symbol interference (ISI) techniques. Some of these techniques are moment space method, characteristic function method, moments method, and the approximate Fourier series method. It has been reported in the literature that these techniques are superior to the central limit theorem-based techniques in approximating the BER but involve higher computational costs. A study of the signal-to-noise ratio (SNR) of Rician fading channels at the correlator receiver's output was done by [7]. The BER performance of the DS-CDMA system in frequency non-selective Rayleigh fading channels for deterministic sequences using the SGA approach was evaluated in [8]. The characteristic function (CF) technique was utilized to assess the performance of the spread spectrum multiple access (SSMA) scheme in an AWGN environment in [9]. The CF method used to evaluate the performance of the DS-SSMA scheme in multipath fading channels with multipath intersymbol interference was applied in [10] without taking into account the MAI effect. An approximate Fourier series technique was utilized in $[11,12]$ to evaluate the BER performance in selective and non-selective Rayleigh fading environments. System degradation caused by an imperfect chip and phase synchronization were also assessed in this technique.

For a given SNR, the BER dependency on the number of users is analyzed in [13], where a closed-form expression for the CF of MAI for asynchronous operation in a Rayleigh fading environment was obtained. An expression for a single integral for overall BER is derived therein.

The conditional CF of MAI together with bounds on the probability error rate for the DS-SSMA scheme are obtained here, whereas [14] derived only the average probability of error at the correlation receiver's output for binary as well as quaternary synchronous and asynchronous DS-SSMA schemes which use a random signature sequence.

The probability density function (PDF) of MAI for a synchronous downlink CDMA in an AWGN case was derived in [15]. This result is then used to derive the conditional probability density function of the MAI, ISI, and noise in multi-carrier code division multiple access scheme (MC-CDMA) provided that the fading environment is known. The PDF of both the MAI and the inter carrier interference is also derived while assuming that the channel-fading effect in CDMA system is known.

A new unified approach to MAI analysis in fading environments was presented in [16], assuming that the channel phase is either known or has been perfectly estimated. Random behavior of the channel fading is also included in [16] to get realistic results for the PDF of MAI and noise. Also, the analysis does not make any simplifying assumptions on the MAI and provides a complete statistical characterization of MAI.

Accurate statistical analysis of MAI in MIMOCDMA systems has not received much attention from researchers, as indicated by the few published studies available in the literature, mainly because the computational complexities it involves. So, to alleviate these complexities, researchers in the past either used some strong assumptions, for example Gaussian assumption for interference in MIMO system [17], or suboptimal approaches to detect the subscriber without involving the need for MAI statistics such as in SIC [18] and PIC [19]. In $[20,21]$, other types of spreading codes are used. These codes, known as chaotic codes, are nonlinear with good correlation properties. In [20], the MAI is analyzed using the Gaussian distribution approximation and in [21] the performance was analyzed under a fading channel scenario in the MIMO case, where in contrast to existing works, an exact characterization of the MAI in a Rayleigh fading environment is developed for MIMO-CDMA systems. Consequently, explicit closed-form expressions for both the PDFs of MAI and MAI plus noise were derived for a Rayleigh fading channel. Recently in [22], the approach of [16] was used to analyze the MAI in MIMOCDMA systems which was later used in [23] to design minimum mean-square-error (MMSE) estimate of fading channels in the presence of MIMO systems. Although, the work in [22] has provided the derivation for the MAI statistics and the work in [23] has provided MMSE estimator design based on this statistics, but there can be many other interesting applications of the derived MAI statistics in practical scenarios which these work failed to provide. In this present work, we aimed to provide more comprehensive treatment of the MAI statistics in MIMOCDMA systems. More importantly, we have provided 
many interesting applications in Section 5 such as the design of optimum receiver, derivation of probability of bit error rate and the derivation for the probability of outage in MIMO-CDMA systems in the presence of Rayleigh flat fading in addition to the design of MMSE estimator. Moreover, we have also highlighted some future research directions/challenges that can be dealt via utilizing the proposed framework of MAI statistics analysis.

\subsection{Main contributions}

In this work, the main contributions are outlined next.

1. Expressions for the PDF of MAI and MAI and noise in MIMO-CDMA systems in the presence of both Rayleigh fading channel and additive Gaussian noise are derived.

2. Three important applications that utilize the newly-derived statistics of MAI are provided and analyzed.

(a) In the first application, an optimum receiver based on ML detection criterion is developed. For this, a new expression for the probability of BER, which is of prime importance in the study of any communication system, is also derived.

(b) In the second application, a new expression for the minimum mean-square-error (MMSE) channel estimation of Rayleigh fading using our derived MAI statistics, is derived.

(c) In the final application, we derive the expression for the probability of outage for MIMO-CDMA systems

3. Although our analysis deals with only a Rayleigh fading channel, our methodology is nevertheless generalized and can be applied to other fading channels.

4. In the last section, we also highlighted some future applications for MIMO-CDMA systems that can employ the proposed framework of designing MAI statistics.

\subsection{Paper organization}

The paper is organized as follows. Section 2 describes the system model used for the analysis. Section 3, where the analysis of the PDF of MAI is carried out is followed by Section 4 which describes the analysis of the PDF of MAIplus-noise. Two important and pertinent applications that utilize the analyses, namely the optimum receiver design using the ML criterion and the MMSE channel estimation, are presented in Section 5. Section 6 presents simulation and numerical results to corroborate the theoretical findings of this work. Finally, Section 7 concludes the paper.

\section{System model}

In this paper, a synchronous DS-CDMA transmitter model for the downlink of a mobile radio network with $N$ transmit and $M$ receive antennas is considered as shown in Fig. 1. Consider a flat-fading channel whose complex impulse response between the $n^{\text {th }}$ transmitter and $m^{\text {th }}$ receiver for the $l^{\text {th }}$ symbol is given by

$$
H_{m n}^{l}(t)=h_{m n}^{l} e^{j \phi_{l}} \delta(t)
$$

where $h_{m n}^{l}$ is the $l^{\text {th }}$ channel tap between the $n^{\text {th }}$ transmitter and the $m^{\text {th }}$ receiver whose value becomes unity for the AWGN channel, and $\phi_{l}$ is the phase of the complex channel for the $l^{\text {th }}$ symbol. Thus, for a Rayleigh fading channel, the PDF of $h_{m n}^{l}$ will be:

$$
f_{h_{m n}^{l}}(x)=\frac{x}{\sigma_{h_{m n}}^{2}} \exp \left(-\frac{x^{2}}{2 \sigma_{h_{m n}}^{2}}\right), \text { for } x>0,
$$

where $\sigma_{h_{m n}}^{2}$ represents the variance of the real and imaginary Gaussian component in the Rayleigh fading. Assuming that the receiver is able to perfectly track the phase of the channel, the detector in the $m^{\text {th }}$ receiver then observes the following signal:

$r_{m}(t)=\sum_{n=1}^{N} \sum_{l=-\infty}^{\infty} \sum_{k=1}^{K} A^{k} b_{n}^{l, k} s_{n}^{l, k}(t) h_{m n}^{l}+\eta_{m}(t), \quad m=1,2, \ldots, M$

where $K$ represents the number of users, $s_{n}^{l, k}(t)$ is the rectangular signature waveform with random $\mathrm{PN}$ signature sequence of the $k^{\text {th }}$ user defined in the $(l-1) T_{b} \leq t \leq$ $l T_{b}, T_{b}$ and $T_{c}$ are the bit period and the chip interval, respectively, related by $N_{c}=T_{b} / T_{c},\left\{b_{n}^{l, k}\right\}$ is the input bit stream of the $k^{\text {th }}$ user, $A^{k}$ is the transmitted amplitude of the $k^{\text {th }}$ user, and $v_{m}$ is the additive white Gaussian noise with zero mean and variance $\sigma_{v}^{2}$ at the $m^{\text {th }}$ receiver. The cross-correlation between the signature sequences of users $j$ and $k$ for the $l^{\text {th }}$ symbol is given by

$$
\rho_{l}^{k, j}=\int_{(l-1) T_{b}}^{l T_{b}} s_{n}^{k}(t) s_{n}^{j}(t) d t=\sum_{i=1}^{N_{c}} c_{l, i}^{k} c_{l, i}^{j}
$$

where $\left\{c_{l, i}^{k}\right\}$ is the normalized spreading sequence (so as to make the autocorrelations of the signature sequences unity) of user $k$ for the $l^{\text {th }}$ symbol. The receiver consists of a matched filter at the front end, which is matched to the signature waveform of the desired user. In our analysis, the desired user will be user 1 . Thus, the matched filter's 


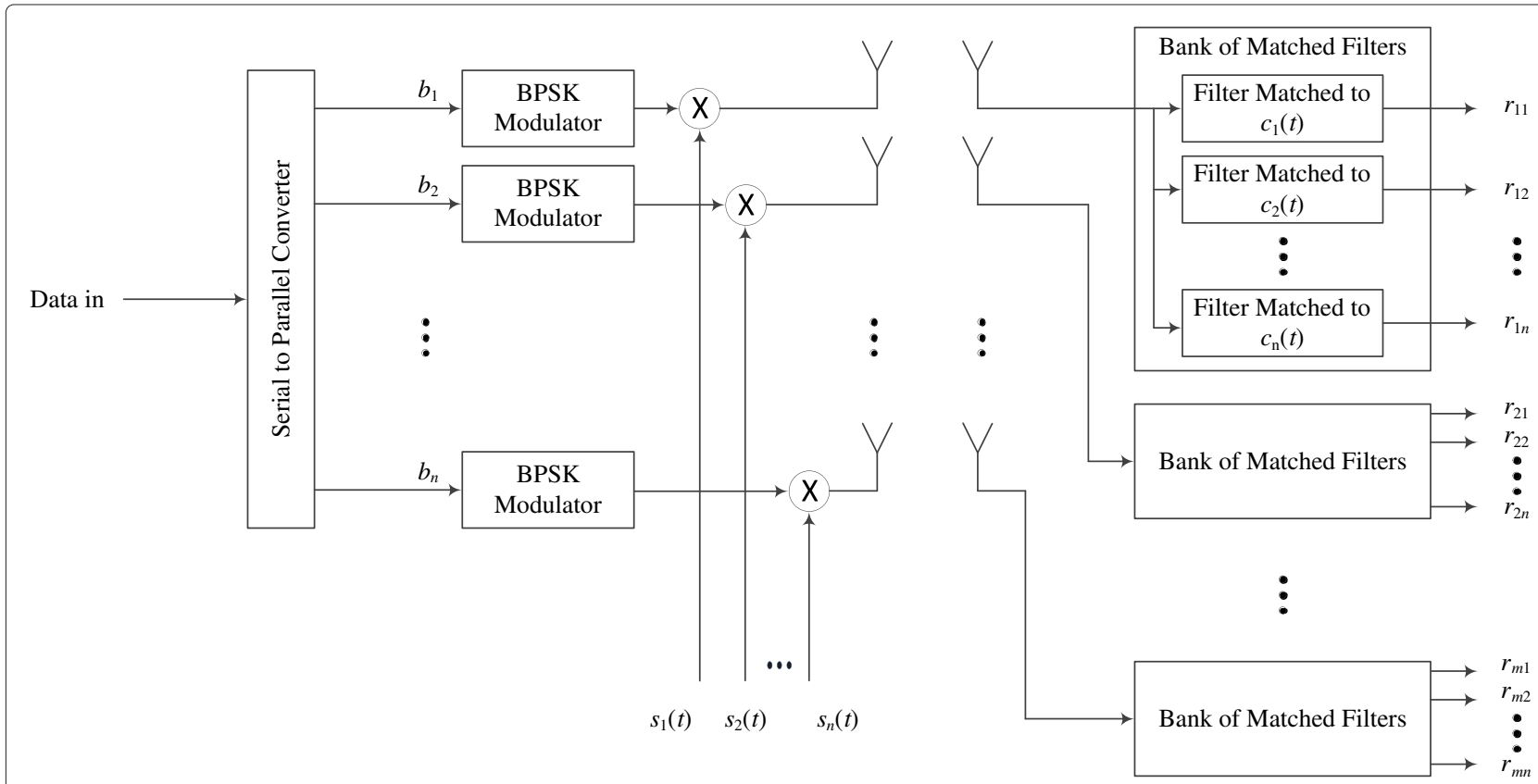

Fig. 1 A block diagram of MIMO-CDMA transmitter and receiver system

output for the $l^{\text {th }}$ symbol at the $m^{\text {th }}$ receiver can be written as follows:

$$
\begin{aligned}
y_{m}^{l} & =\int_{(l-1) T_{b}}^{l T_{b}} r_{m}(t) s_{m}^{l, 1}(t) d t \\
& =\sum_{n=1}^{N} A^{1} b_{n}^{l, 1} h_{m n}^{l}+z_{m}^{l}+\eta_{m}, \quad m=1,2, \ldots M
\end{aligned}
$$

where $z_{m}^{l}$ is the MAI at the $m^{\text {th }}$ receiver for the $l^{\text {th }}$ symbol in the presence of the fading channel $h_{m n}^{l}$ and is given by

$$
z_{m}^{l}=\sum_{n=1}^{N} \sum_{k=2}^{K} A^{k} b_{n}^{l, k} \rho_{n}^{k, 1} h_{m n}^{l}, \quad m=1,2, \ldots M
$$

which can also be expressed as follows

$$
z_{m}^{l}=\sum_{n=1}^{N} I_{m n}^{l} h_{m n}^{l}, \quad m=1,2, \ldots, M
$$

where the random variable $I_{m n}^{l}=\sum_{k=2}^{K} A^{k} b_{n}^{l, k} \rho_{n}^{k, 1}$, which is nothing but MAI in AWGN environment, is shown to follow Gaussian behavior in [16], that is, $I_{m n}^{l} \sim \mathcal{N}\left(0, \sigma_{I}^{2}\right)$ where $\sigma_{I}^{2}=\frac{A^{2}(K-1)}{N_{c}}$. For the sake of tractability of the analysis, we have assumed the same behavior for random variable $I_{m n}^{l}$ in the MIMO system.

\section{PDF of MAI in MIMO-CDMA systems}

In order to find the PDF of the random variable $U_{n}^{l}=$ $I_{m n}^{l} h_{m n}^{l}$, we use the assumption of statistical independence of $I^{l, k}$ and $h_{m n}^{l}$ as follows

$$
\begin{aligned}
f_{U}(u)= & \int_{-\infty}^{\infty} \frac{1}{|x|} f_{I}\left(\frac{u}{x}\right) f_{h_{m n}^{l}}(x) d x, \quad x>0 \\
= & \int_{0}^{\infty} \frac{1}{x} \frac{1}{\sqrt{2 \pi \sigma_{I}^{2}}} \exp \left(-\frac{u^{2}}{2 x^{2} \sigma_{I}^{2}}\right) \frac{x}{\sigma_{h_{m n}}^{2}} \\
& \times \exp \left(-\frac{x^{2}}{2 \sigma_{h_{m n}^{2}}^{2}}\right) d x,
\end{aligned}
$$

which is found to be

$$
f_{U}(u)=\frac{1}{2 \sigma_{I} \sigma_{h_{m n}}} \exp \left(-\frac{|u|}{\sigma_{h_{m n}} \sigma_{I}}\right)
$$

The characteristic function of the random variable $U_{n}^{l}$ can be then be evaluated as

$$
\begin{aligned}
\Phi_{U_{n}}(\omega) & =E\left[e^{i w U}\right] \\
& =\frac{1}{2 \sigma_{I} \sigma_{h_{m n}}} \int_{-\infty}^{\infty} \exp (i \omega u) \exp \left(-\frac{|u|}{\sigma_{h_{m n}} \sigma_{I}}\right) d u \\
\Phi_{U_{n}}(\omega) & =\frac{1}{\omega^{2} \sigma_{U_{n}}^{2}+1}
\end{aligned}
$$

where $^{1} \sigma_{U_{n}}^{2}=\sigma_{I}^{2} \sigma_{h_{m n}}^{2}$. Since the interferers are assumed to be independent of, but not identical to, each other, it can therefore be observed from (7) that the characteristic function of $z_{m}^{l}$ will be the product of $N$ characteristic functions of the independent random variables $U_{n}^{l}$, that is,

$$
\Phi_{Z}(\omega)=\prod_{n=1}^{N} \Phi_{U_{n}}(\omega)=\prod_{n=1}^{N} \frac{1}{\omega^{2} \sigma_{U_{n}}^{2}+1}
$$


Inverse-Fourier transforming the above characteristic function will yield:

$$
\begin{aligned}
f_{Z}(z) & =\mathcal{F}^{-1}\left[\Phi_{Z}(\omega)\right] \\
& =\frac{1}{2 \pi} \int_{-\infty}^{\infty} \exp (-i \omega z) \prod_{n=1}^{N} \frac{1}{\omega^{2} \sigma_{U_{n}}^{2}+1} d \omega
\end{aligned}
$$

The above integral is evaluated in Appendix A for two different scenarios: (1) $\sigma_{U_{n}}^{2}$ have distinct values for each $n$ and (2) $\sigma_{U_{n}}^{2}$ are equal for all $n$. As a result, expression for the PDF of $z$ can be shown to

$$
f_{Z}(z)= \begin{cases}\sqrt{\frac{2|z|}{\pi \sigma_{U}}} \frac{1}{\left(2 \sigma_{U}\right)^{N}} \frac{(|z|)^{N-1}}{\Gamma(N)} K_{N-\frac{1}{2}}\left(\frac{|z|}{\pi \sigma_{U}}\right) & \text { for identical } \sigma_{U_{n}}^{2}, \\ \frac{1}{2} \sum_{n=1}^{N} \frac{C_{n} e^{-\frac{z}{\sigma_{U_{n}}}}}{\sigma U_{U_{n}}} & \text { for distinct } \sigma_{U_{n}}^{2} .\end{cases}
$$

where $C_{n}$ is defined in (59) (see Appendix A) and $K_{\mu}(z)$ is the Bessel function for imaginary arguments [24] and it is defined in (67). The PDF of MAI in (12) for the distinct scenario shows that the MAI experienced at any receiving antenna is a sum of Laplacian distributed random variables. It can be easily seen that by setting $N=1$ in the above, the PDF of MAI will reduce to a single Laplacian random variable, which is consistent with the result obtained in [16].

\subsection{Variance of MAI}

The MAI term is already defined in (7). Since it is assumed that the interferers are independent and zero mean (zero mean is due to the fact that $E\left[I_{m n}^{l}\right]=0$ ), the variance of total MAI in MIMO-CDMA system (denoted by $\sigma_{z}^{2}$ ) can be evaluated as follows:

$$
\sigma_{z}^{2}=\sum_{n=1}^{N} E\left[\left(I_{m n}^{l}\right)^{2}\right] E\left[\left(h_{m n}^{l}\right)^{2}\right]=\sum_{n=1}^{N} 2 \sigma_{I}^{2} \sigma_{h_{m n}}^{2}
$$

In the special case when $\sigma_{h_{m n}}^{2}=\sigma_{h}^{2}$ for all $m$ and $n$, we get

$$
\sigma_{z}^{2}=2 N \sigma_{I}^{2} \sigma_{h}^{2}
$$

\section{PDF of MAI-plus-noise}

In order to find the PDF of the MAI-plus-noise, we consider $\kappa_{m}^{l}$ to be a random variable resulting in MAI-plusnoise, that is,

$$
\kappa_{m}^{l}=z_{m}^{l}+\eta_{m}^{l} \quad m=1,2, \ldots, M
$$

where $\eta_{m}^{l}$ is the AWGN part. Thus, the PDF of the random variable $\kappa_{m}^{l}$ can be evaluated by the convolution of the PDFs of $z_{m}^{l}$ and $\eta_{m}^{l}$ as follows:

$$
\begin{aligned}
f_{\kappa_{m}^{l}}(\kappa) & =f_{Z}(z) * f_{\eta}(\eta) \\
& =\int_{-\infty}^{\infty} f_{Z}(t) f_{\eta}(\kappa-t) d t \\
& =\sum_{j=1}^{N} \frac{C_{n}}{2 \sigma_{U_{n}}} \beta_{n}
\end{aligned}
$$

where $\beta_{n}$ is the integral defined as

$$
\beta_{n}=\frac{1}{\sqrt{2 \pi} \sigma_{\eta}} \int_{-\infty}^{\infty} \exp \left(-\frac{|t|}{\sigma_{U_{n}}}-\frac{(\kappa-t)^{2}}{2 \sigma_{\eta}^{2}}\right) d t
$$

and can be evaluated using the technique of "completing the square" and by employing the definition of the error complement function $\operatorname{erfc}(\mathrm{x})$ [24]. This yields

$$
\begin{aligned}
\beta_{n}= & \frac{e^{\frac{\sigma_{\eta}^{2}}{2 \sigma_{U_{j}}^{2}}}}{2} e^{\frac{-\kappa}{\sigma U_{n}}} \operatorname{erfc}\left(\frac{\sigma_{\eta}}{\sqrt{2} \sigma_{U_{n}}}-\frac{\kappa}{\sqrt{2} \sigma_{\eta}}\right) \\
& +e^{\frac{\kappa}{\sigma_{U_{n}}}} \operatorname{erfc}\left(\frac{\sigma_{\eta}}{\sqrt{2} \sigma_{U_{n}}}+\frac{\kappa}{\sqrt{2} \sigma_{\eta}}\right)
\end{aligned}
$$

Now, using the fact that

$$
\begin{aligned}
\Gamma\left(\frac{1}{2}, x ; b\right)= & \frac{\sqrt{\pi}}{2} \exp (-2 \sqrt{b}) \operatorname{erfc}(\sqrt{x}-\sqrt{b / x}) \\
& +\exp (2 \sqrt{b}) \operatorname{erfc}(\sqrt{x}+\sqrt{b / x})
\end{aligned}
$$

where

$$
\Gamma(a, x ; b):=\int_{x}^{\infty} t^{a-1} \exp (-t-b / t) d t .
$$

is the generalized incomplete gamma function [25].

We can express the $\operatorname{PDF} f_{\kappa_{m}^{l}}(\kappa)$ as

$$
f_{\kappa_{m}^{l}}(\kappa)=\frac{1}{2 \sqrt{\pi}} \sum_{n=1}^{N} \mathcal{A}_{n} \Gamma\left(\frac{1}{2}, \mathcal{B}_{n} ; \frac{\kappa^{2}}{\mathcal{C}_{n}}\right)
$$

where, for compactness purposes, the symbols $\mathcal{A}_{n}=\frac{C_{n}}{\sigma U_{n}}$ $\exp \left(\mathcal{B}_{n}\right), \mathcal{B}_{n}=\frac{\sigma_{n}^{2}}{2 \sigma_{U_{n}}^{2}}$ and $\mathcal{C}_{n}=4 \sigma_{U_{n}}^{2}$ have been introduced.

\section{Important applications involving the utilization of the exact MAI statistics}

As stated earlier, MAI introduces an overhead on CDMA systems, which acts as a limiting factor in system performance. It is evident from previous work that the performance analysis of CDMA systems has not really 
utilized the exact statistics of MAI. Therefore, system designs based on approximated MAI statistics have lead to poor system performance. In this work, in order that the knowledge of the exact MAI statistics does indeed remarkably improve system performance, we present here two important applications to emphasize this point. These applications are listed below and discussed in the ensuing subsections:

- design of an optimum MIMO-CDMA receiver using the ML criterion.

- design of MMSE channel estimator for Rayleigh fading in MIMO-CDMA systems.

- derivation of probability of outage for Rayleigh fading in MIMO-CDMA systems.

\subsection{Design of an optimum MIMO-CDMA receiver using the ML criterion}

Both the design and characteristics of optimum receivers in the presence of AWGN for different modulation methods have been extensively covered in the literature [26]. It is reported in the literature that the optimum detector for a AWGN channel is comprised of a correlation demodulator or a matched filter followed by an optimum decision rule, and is based on the maximum a posteriori probability (MAP) criterion when the a priori probabilities of the transmitted signal are unequal, and the ML criterion when these a priori probabilities are equal. Decisions based on any of the criteria used depend on the conditional PDF of the received vector at the output of either the matched filter or the correlator, whichever is used. In our analysis, the ML criterion is utilized, since we are using the case where the a priori probabilities of the transmitted signal are equal.

BER is defined as the number of bits received in error with respect to the total number of bits received at a particular receiver. BER performance is considered to be a very important criterion for CDMA systems as it determines the quality of transmission as well as the amount of data that can be transmitted per unit of bandwidth. Since all users contribute to the interference levels at the receiving side, the BER of each user increases when several users try to access the same channel simultaneously. Subsequently, the maximum number allowable of users is determined by the amount of interference which can be tolerated [27]. This section deals with the design of an optimum receiver in the presence of MAI-plus-noise for a Rayleigh fading environment. The probability of error is derived here for the maximum likelihood receiver and the simulation results obtained here support our analytical finding.

At the front end of the optimum receiver, a matched filter is placed, and is matched to the desired subscriber, which in our case, is user 1 . The output of the matched filter that is matched to the signature waveform of the desired user for the $l$ th symbol can be written as

$$
y_{m}^{l}=w_{m, i}^{l}+\kappa_{m}^{l} \quad i=1,2 \quad \text { (for BPSK signals) }
$$

where $w_{m, i}^{l}$ is the desired symbol in a MIMO system. If $E_{b}$ represents the energy-per-bit, then $w_{m, i}^{l}$ is given by either $+N \alpha_{i} \sqrt{E_{b}}$ or $-N \alpha_{i} \sqrt{E_{b}}$ for BPSK signals. From the output of the matched filter given in (5), the desired signal is given by $\sum_{n=1}^{N} A^{1} b_{n}^{l, 1} h_{m n}^{l}$, where the channel taps $\alpha_{i}$ are no longer individual Rayleigh fading contributions but are rather a sum of all Rayleigh fading contributions. This can be seen as

$$
\sum_{n=1}^{N} A^{1} b_{n}^{l, 1} h_{m n}^{l}=A^{1} b^{l, 1} \sum_{n=1}^{N} h_{m n}^{l}
$$

where we are assuming that the same data is being transmitted from each antenna for a given user (i.e., $b_{n}^{l, 1}=$ $\left.b^{l, 1} \forall n\right)$. Thus, $\alpha^{l}=\sum_{n=1}^{N} h_{m n}^{l}$ represents the sum of $N$ independent Rayleigh random variables whose PDF can be obtained from ([28] Eq. 4a-b) as

$$
f_{\alpha}(\alpha)=\frac{\alpha^{2 N-1} e^{-\frac{\alpha^{2}}{2 \psi}}}{2^{N-1} \psi^{N}(N-1) !}
$$

where $\psi=\frac{\sigma^{2}}{N}[(2 N-1) ! !]^{1 / N},(2 N-1) ! !=(2 N-1)$ $(2 N-3) \cdots 3.1, \alpha=x / \sqrt{N}, x$ is the normalized Rayleigh random variable and $N$ is the number of transmitters.

For BPSK signaling, the conditional PDF $p\left(y_{m}^{l} \mid w_{m, 1}^{l}\right)$ can be obtained by using (20) as

$p\left(y_{m}^{l} \mid w_{m, 1}^{l}\right)=\frac{1}{2 \sqrt{\pi}} \sum_{n=1}^{N} \mathcal{A}_{n} \Gamma\left(\frac{1}{2}, \mathcal{B}_{n} ; \frac{\left(y_{m}^{l}-N \alpha_{i} \sqrt{E_{b}}\right)^{2}}{\mathcal{C}_{n}}\right)$

For the case when $w_{m, 1}^{l}$ and $w_{m, 2}^{l}$ have equal a priori probabilities, then according to the ML criterion, the optimum test statistic is the likelihood ratio $\left(\Lambda=p\left(y_{m}^{l} \mid w_{m, 1}^{l}\right) / p\left(y_{m}^{l} \mid w_{m, 2}^{l}\right)\right)$. Now assuming that the channel attenuation $\left(\alpha^{l}\right)$ is deterministic, therefore any transmission error that has occurred is only due to the MAI-plus-noise contribution term $\left(Z_{m, i}^{l}\right)$. It is shown in [16] that this MAI-plus noise term has a zero mean and a zero skewness, which shows its symmetric behavior about its mean. Consequently, the conditional PDF $p\left(y_{m}^{l} \mid w_{m, 1}^{l}\right)$ with deterministic channel attenuation will also be symmetric, thus resulting in the threshold for the ML optimum receiver being its mean value of zero. Finally, the probability of error, given that $w_{m, 1}^{l}$ is transmitted, is found to be: 


$$
\begin{aligned}
P\left(e \mid w_{i, 1}\right) & =\int_{-\infty}^{0} p\left(y_{m}^{l} \mid w_{m, 1}^{l}\right) d y_{m}^{l} \\
& =\frac{1}{2 \sqrt{\pi}} \sum_{n=1}^{N} \int_{-\infty}^{0} \mathcal{A}_{n} \Gamma\left(\frac{1}{2}, \mathcal{B}_{n} ; \frac{\left(y_{m}^{l}-N \alpha_{i} \sqrt{E_{b}}\right)^{2}}{\mathcal{C}_{n}}\right) d y_{m}^{l} \\
& =\frac{1}{2} \sum_{n=1}^{N} C_{n} \exp \left(\frac{\sigma_{\eta}^{2}}{2 \sigma_{I}^{2} \sigma_{\alpha}^{2}}\right) \int_{\sigma_{\eta}^{2} / 2 \sigma_{I}^{2} \sigma_{\alpha}^{2}}^{\infty} e^{-t} \operatorname{erfc}\left(\sqrt{\frac{\alpha^{2} N^{2} E_{b}}{4 \sigma_{I}^{2} \sigma_{\alpha}^{2} t}}\right) d t
\end{aligned}
$$

where we have replaced the constants $\mathcal{A}_{n}, \mathcal{B}_{n}$, and $\mathcal{C}_{n}$ in (25) by their respective expressions mentioned right after (20). In the derivation of BER, we require the PDF of $\alpha^{2}$ for which we need the PDF of $\alpha$. In order to get the PDF of $\alpha^{2}$, we need to apply a transformation to the random variable $\alpha$ to get the PDF of the squared one. This carried out as follows: We define a new random variable

$$
\gamma_{\kappa}=\frac{\alpha^{2} N^{2} E_{b}}{4 \sigma_{I}^{2} \sigma_{\alpha}^{2} t}
$$

The mean of $\gamma_{\kappa}$, i.e., $\overline{\gamma_{\kappa}}=E\left[\gamma_{\kappa}\right]$ can be found as

$$
\overline{\gamma_{\kappa}}=E\left[\gamma_{\kappa}\right]=\frac{E\left[\alpha^{2}\right] N^{2} E_{b}}{4 \sigma_{I}^{2} \sigma_{\alpha}^{2} t}=\frac{2 \psi N N^{2} E_{b}}{4 \sigma_{I}^{2} \sigma_{\alpha}^{2} t}=\frac{\psi N^{3} E_{b}}{2 \sigma_{I}^{2} \sigma_{\alpha}^{2} t}
$$

From (27) we have

$$
\alpha=\frac{2}{N} \sqrt{\frac{\gamma_{\kappa} \sigma_{I}^{2} \sigma_{\alpha}^{2} t}{E_{b}}}
$$

So the PDF of $\gamma_{\kappa}$ can be found by the transformation method as

$$
f_{\gamma_{\kappa}}\left(\gamma_{\kappa}\right)=\left.\frac{f_{\alpha}(\alpha)}{\left|d \gamma_{\kappa} / d \alpha\right|}\right|_{\alpha=\frac{2}{N} \sqrt{\frac{\gamma_{\kappa} \sigma_{I}^{2} \sigma_{\alpha}^{2} t}{E_{b}}}}
$$

where $\frac{d \gamma}{d \alpha}$ can be found by differentiating (27) to get

$$
\frac{d \gamma}{d \alpha}=\frac{\alpha N^{2} E_{b}}{2 \sigma_{I}^{2} \sigma_{\alpha}^{2} t}=\frac{2}{N} \sqrt{\frac{\gamma_{\kappa} \sigma_{I}^{2} \sigma_{\alpha}^{2} t}{E_{b}}} \times \frac{N^{2} E_{b}}{2 \sigma_{I}^{2} \sigma_{\alpha}^{2} t}
$$

The expression for the BER is evaluated in Appendix A and can be setup as

$$
P(e)=\frac{1}{2} \sum_{j=1}^{N} C_{j} 2^{1-N} \exp \left(\frac{\sigma_{\eta}^{2}}{2 \sigma_{I}^{2} \sigma_{\alpha}^{2}}\right)\left(\frac{\sigma_{I}^{2} \sigma_{\alpha}^{2}}{\psi N^{2} E_{b}}\right)^{N} \frac{\Gamma(2 N)}{\Gamma(N+1)} \times I_{j}
$$

where

$I_{j}=\int_{\sigma_{\eta}^{2} / 2 \sigma_{I}^{2} \sigma_{\alpha}^{2}}^{\infty} e^{-t} t^{N}{ }_{2} F_{1}\left(N, N+\frac{1}{2} ; N+1,-\frac{2 \sigma_{I}^{2} \sigma_{\alpha}^{2} t}{\psi N^{2} E_{b}}\right) d t$

There is no closed-form expression for the above integral and so it was evaluated numerically.

\subsection{Design of MMSE channel estimator for Rayleigh fading in MIMO-CDMA systems}

In order to ascertain the impact of the above analysis from an application perspective, a pilot-aided MIMO channel estimation scheme is devised which extends the original work done in [29]. The estimation is based on the MMSE criterion. Taking the sum of Rayleigh fading in the MIMO system for the $l^{\text {th }}$ symbol to be $\alpha^{l}=\sum_{n=1}^{N} h_{m n}^{l}$, we define the signal of the desired user as

$$
x \triangleq b^{1} \alpha
$$

where we have dropped the time index for simplicity. Denoting the estimate of $x$ by $\hat{x}$, the mean-square-error cost function for the MMSE estimate is

$$
J=E\left[(x-\hat{x})^{2}\right]
$$

The solution of the cost function in Eq. (35) is well known and is given by [30]

$$
\hat{x}(y)=E[x \mid y]=\int_{-\infty}^{\infty} x f_{x \mid y}(x \mid y) d x
$$

The estimate of $\hat{x}$ will lead us to the estimate of $\hat{\alpha}$ from (34)

$$
\hat{\alpha}=\frac{\hat{x}}{b^{1}}
$$

In order to evaluate $\hat{x}$, we require the conditional PDF $f_{x \mid y}(x \mid y)$, which can be obtained, using Bayes theorem as [30]

$$
f_{x \mid y}(x \mid y)=\frac{f_{y \mid x}(y \mid x) f_{x}(x)}{f(y)}
$$

To use (38), we need to evaluate first $f_{x}(x)$. Since the random variable $x$ is the product of two random variables $b^{1}$ and $\alpha$, its PDF can be evaluated using the procedure of the random variable transformation as follows:

$$
f_{x}(x)=\int_{0}^{\infty} \frac{1}{\lambda} f_{\alpha}(\lambda) f_{b}\left(\frac{x}{\lambda}\right) d \lambda
$$

where $f_{b}(b)=0.5[\delta(b+1)+\delta(b-1)]$ and $f_{\alpha}(\alpha)$ is the PDF of the sum of Rayleigh fading random variables given in (23). Inserting both PDFs in (39), the integral results in

$$
f_{x}(x)=\frac{\mathcal{G}}{\psi^{N}} x^{2 N-1} \exp \left(-\frac{x^{2}}{2 N \psi}\right)
$$


where $\mathcal{G}=\left(\frac{1}{\sqrt{N}}\right)^{2 N}\left(\frac{1}{2}\right)^{N} \frac{1}{(N-1) !} x^{2 N-1}$. The conditional PDF of $f_{y \mid x}(y \mid x)$, which can be found by (20), is given by

$$
f_{y \mid x}(y \mid x)=\frac{1}{2 \sqrt{\pi}} \sum_{n=1}^{N} \mathcal{A}_{n} \Gamma\left(\frac{1}{2}, \mathcal{B}_{n} ; \frac{(y-x)^{2}}{\mathcal{C}_{n}}\right)
$$

Using the fact that $\forall y$

$$
f(y)=\int_{-\infty}^{\infty} f_{y \mid x}(y \mid x) f_{x}(x) d x
$$

Plugging both (40) and (41) in (42) yields

$$
f(y)=\frac{1}{2 \sqrt{\pi}} \frac{\mathcal{G}}{\psi^{N}} \sum_{n=1}^{N} \mathcal{A}_{n} I_{n(1)}
$$

where $I_{n(1)}$ is given by

$$
I_{n(1)}=\int_{-\infty}^{\infty} \Gamma\left(\frac{1}{2}, \mathcal{B}_{n} ; \frac{(y-x)^{2}}{\mathcal{C}_{n}}\right) \exp \left(-\frac{x^{2}}{2 N \psi}\right) d x
$$

Substituting the PDFs of $f(y)$ and $f_{y \mid x}(y \mid x)$ in (38) and using (36) through some mathematical simplification, we get

$$
\hat{x}=\sum_{n=1}^{N} \frac{I_{n(2)}}{I_{n(1)}}
$$

where $I_{n(2)}$ is given by

$$
I_{n(2)}=\int_{-\infty}^{\infty} x^{2 N} \Gamma\left(\frac{1}{2}, \mathcal{B} ; \frac{(y-x)^{2}}{\mathcal{C}}\right) \exp \left(-\frac{x^{2}}{2 N b}\right) d x
$$

Although the integrals in (44) and (46) cannot be evaluated analytically, there are various numerical integration techniques to evaluate these numerically [31]. Hence, the estimate in (45) can be used in (37) to get the MMSE estimate of the Rayleigh fading channel for MIMO-CDMA system.

\section{Outage probability}

It is well known that outage probability is used as a performance measure for a communication link when signal to interference-plus-noise ratio (SINR) is a random quantity. This happens when we deal with random channel as in the case fading.

The outage probability is the probability that the received SINR is below certain threshold (corresponding to a minimum required rate) in a given duration. Thus, the outage probability (denoted by $P_{\text {out }}$ ) can be expressed as

$$
P_{\text {out }}=\operatorname{Pr}[\operatorname{SINR} \leq \zeta]
$$

where $\zeta$ is a certain threshold to meet the required minimum rate. In our case, the instantaneous SINR can be expressed as

$$
\operatorname{SINR}=\frac{S}{\sigma_{z}^{2}+\sigma_{\eta}^{2}}
$$

where $S=\left(\sum_{n=1}^{N} A^{1} b_{n}^{l, 1} h_{m n}^{l}\right)^{2}$ represents the instantaneous desired signal power which make the SINR a random quantity. Here, $\sigma_{z}^{2}=E\left[\left(z_{m}^{l}\right)^{2}\right]$ and $\sigma_{\eta}^{2}=E\left[\left(\eta_{m}^{l}\right)^{2}\right]$ represent the MAI power and the noise power, respectively. In order to evaluate the MAI power, we make use of the probability of MAI expression derived in (12). Thus, we can evaluate $\sigma_{z}^{2}$ as

$$
\sigma_{z}^{2}=\int_{-\infty}^{\infty} z^{2} f_{Z}(z) d z=\int_{-\infty}^{\infty} z^{2} \frac{1}{2} \sum_{n=1}^{N} \frac{C_{n} e^{-\frac{|z|}{\sigma_{U_{n}}}}}{\sigma_{U_{n}}} d z
$$

Since, the PDF of MAI is even symmetric due to the term $|z|$, we can simplify the above as

$$
\sigma_{z}^{2}=\sum_{n=1}^{N} \frac{C_{n}}{\sigma_{U_{n}}} \int_{0}^{\infty} z^{2} \sum_{n=1}^{N} e^{-\frac{z}{\sigma U_{n}}} d z
$$

Now, by employing a change of variable with $t=-\frac{z}{\sigma_{U_{n}}}$, we can set up the above as

$$
\sigma_{z}^{2}=\sum_{n=1}^{N} \sigma_{U_{n}}^{2} C_{n} \int_{0}^{\infty} t^{2} e^{-t} d t
$$

Next, by using the definition of gamma function $\Gamma(\alpha)=$ $\int_{0}^{\infty} t^{\alpha-1} e^{-t} d t$ and knowing the fact that $\Gamma(1)=1$, we conclude that

$$
\sigma_{z}^{2}=\sum_{n=1}^{N} \sigma_{U_{n}}^{2} C_{n}
$$

Now, we turn our attention to characterize the instantaneous desired signal power (S). To do so, we first utilize the formulation given in (22) which allows us to write $S$ as

$$
S=\left(A^{1} b^{l, 1}\right)^{2}\left(\sum_{n=1}^{N} h_{m n}^{l}\right)^{2}
$$

Since we are focusing on BPSK modulation, we have $\left(A^{1} b^{l, 1}\right)^{2}=1$. As a result, the random variable $S$ can be formulated as $S=\alpha^{2}$ where $\alpha$ is the sum of $N$ independent Rayleigh random variables whose PDF is given in (23). Eventually, this provides the following PDF of random variable $S$

$$
f_{S}(s)=\frac{s^{N-1} e^{-\frac{s}{2 \psi}}}{2^{N} \psi^{N}(N-1) !}
$$


Outage probability is given by

$$
\begin{aligned}
P_{\text {out }} & =\int_{0}^{\zeta\left(\sigma_{z}^{2}+\sigma_{\eta}^{2}\right)} f_{S}(s) d s \\
& =\int_{0}^{\zeta\left(\sigma_{z}^{2}+\sigma_{\eta}^{2}\right)} \frac{s^{N-1} e^{-\frac{s}{2 \psi}}}{2^{N} \psi^{N}(N-1) !} d s
\end{aligned}
$$

By using the change of variable $t=\frac{s}{2 \psi}$, we get

$$
P_{\text {out }}=\frac{1}{(N-1) !} \int_{0}^{\frac{\zeta\left(\sigma_{z}^{2}+\sigma_{\eta}^{2}\right)}{2 \psi}} t^{N-1} e^{-t} d t
$$

Finally, by utilizing the definition of incomplete gamma function (i.e., $\gamma(\alpha, x) \triangleq \int_{0}^{x} t^{\alpha-1} e^{-t} d t$ ), we obtain

$$
P_{\text {out }}=\frac{1}{(N-1) !} \gamma\left(N, \frac{\zeta\left(\sigma_{z}^{2}+\sigma_{\eta}^{2}\right)}{2 \psi}\right)
$$

\section{Simulation results}

In this section, we present some simulation results to validate our theoretical findings. The simulation setup consists of two different scenarios of $2 \times 2$ and $4 \times 4$ MIMO systems. The CDMA system used here relies on the use of random signature sequences of length 31 . The waveform chosen for the PN signatures are rectangular chip waveforms. The channel noise is taken to be an additive white Gaussian noise with an SNR of $20 \mathrm{~dB}$. The Rayleigh channel is chosen to be flat and slow-fading. The simulation results are discussed according to the various tasks in the ensuing subsections.

\subsection{Investigation on MAl statistics}

In this section, we aim to investigate the effect of different parameters on the PDF of the MAI and MAI-plusnoise, namely the effect of the number of transmitting and receiving antennas, length of the pseudo-randomnoise $(\mathrm{PN})$ sequence used, and the number of users in the system. Moreover, we validate the theoretically derived results for the PDF of MAI and MAI-plus-noise by comparing them with various simulation experiments.

Figure 2 shows the effect of system's diversity order on the PDF of MAI. As can be seen, the variance of MAI increases with an increase in diversity. This is consistent with our theoretical predictions in that the severity of the interference would increase in the presence of such diversity because the cumulative effect of all the interferences emerging from the various transmitting channels. The effect of the length of the signature sequence on the PDF of MAI can be seen in Fig. 3 where the MIMO system used is a $4 \times 4$ one. This is also consistent with the results reported in [16]. The variance of MAI decreases with an increase in the length of the PN sequence.

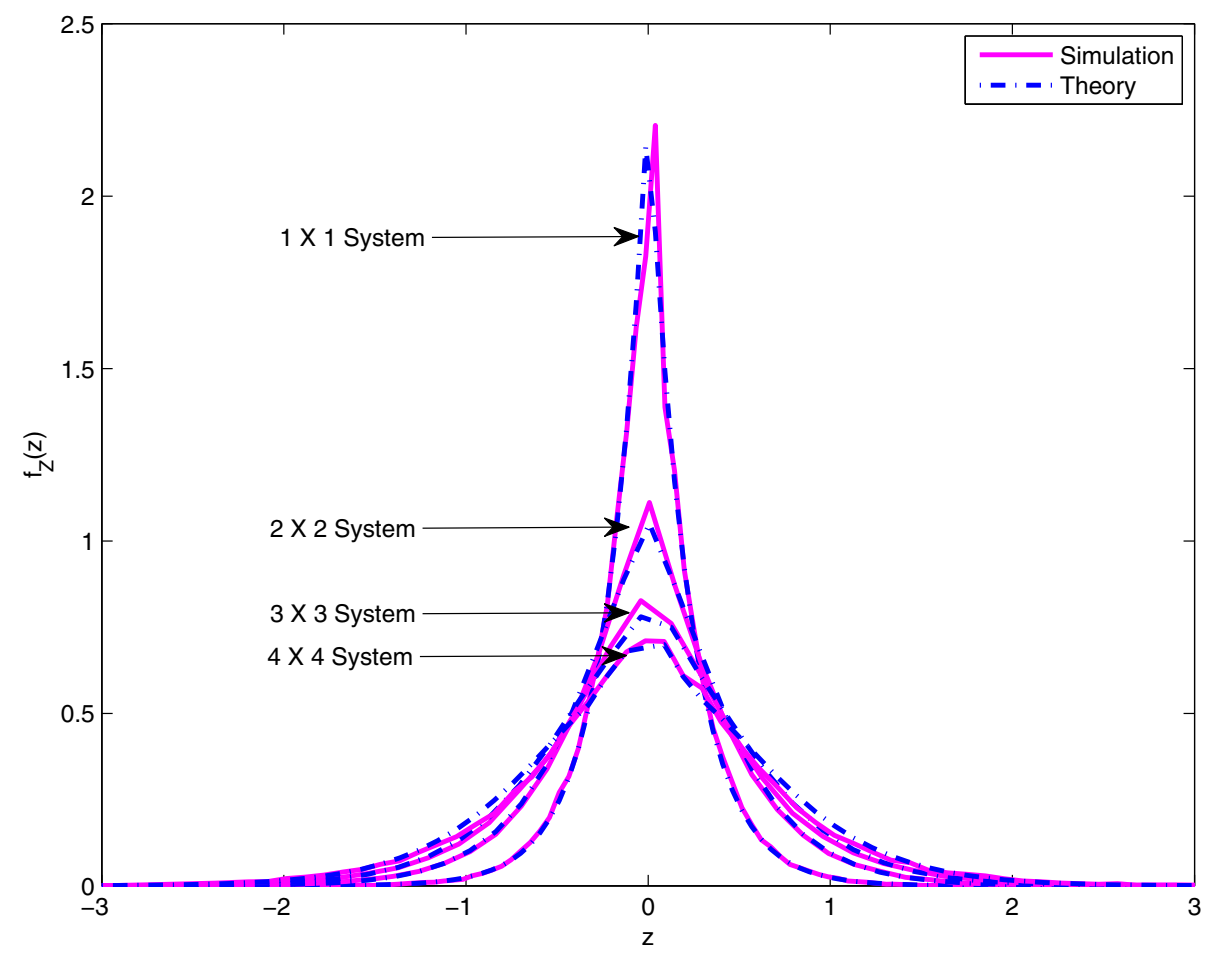

Fig. 2 PDF of MAI for different scenarios of transmit and receive antennas 


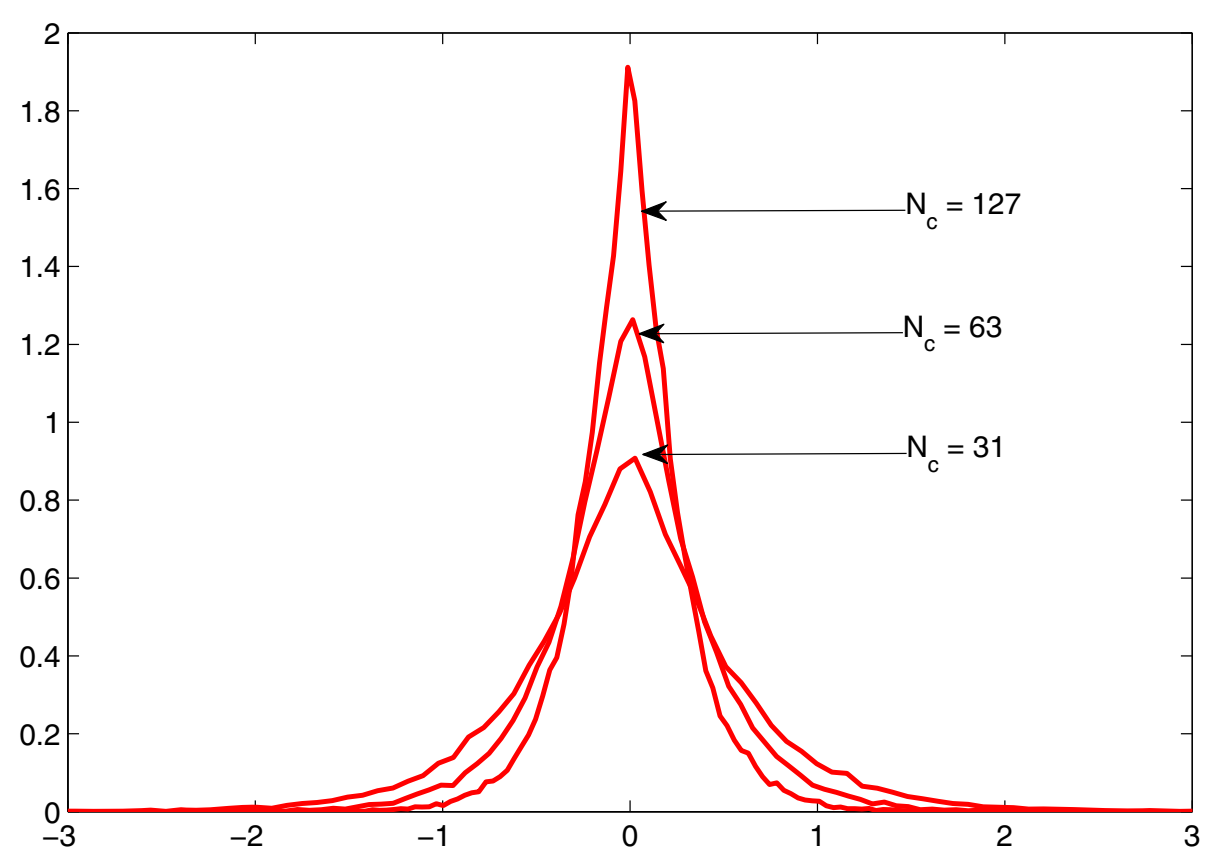

Fig. 3 PDF of MAI for $4 \times 4$ MIMO system under various lengths of PN sequences

Table 1 shows the comparison between the analytical and experimental variances in each case, which corroborates our theoretical analysis. As shown in our analysis, the MIMO-MAI is a sum of Laplacian-distributed random variables. Thus, to ascertain this fact, it is necessary to test the kurtosis of the MIMO-MAI. Table 1 lists the kurtosis of the MAI which is gradually increasing as the signature length increases. The decrease in the signature sequence's length would result in MAI in AWGN becoming Gaussian while affecting the MAI under the fading environment at the same time. Although from simulation, it cannot be predicted whether the MIMO-MAI would exhibit any Gaussian behavior. However, it can be safely assumed that for a short-enough signature length, the MIMO-MAI would assume a Gaussian behavior. This fact is also observed in Fig. 3.

Figure 4 shows the effects of interference on both the PDF of MAI-plus-noise and on the system's performance. It is intuitive to predict that the system will be severely degraded by an increase in the number of users. As the number of users increases, the power of the MAI

Table 1 Kurtosis and variance of MAI in a $4 \times 4$ MIMO system with $K=10$

\begin{tabular}{lllll}
\hline & $N_{C}=31$ & $N_{C}=63$ & $N_{C}=127$ & $N_{C}=255$ \\
\hline Experimental kurtosis of MAl & 4.02 & 4.11 & 4.16 & 4.21 \\
Experimental variance & 1.1689 & 0.5736 & 0.2823 & 0.1411 \\
Analytical variance & 1.1613 & 0.5714 & 0.2835 & 0.1412 \\
\hline
\end{tabular}

experienced by the desired user will also increase as depicted in Fig. 4. Table 2 lists the experimental kurtosis of MAI-plus-noise under different capacity and diversity scenarios to test the Gaussianity assumption. In all the cases studied, the kurtosis increases with an increase in the system's user capacity. Particularly for the $2 \times 2$ MIMO case, the MAI-plus-noise behaves more like a Gaussian signal as its kurtosis gets closer to that of a Gaussian distribution which is 3 . Moreover, it can be seen that the kurtosis decreases with an increase in number of users. For example, in case of $2 \times 2$ MIMO with 20 users, it reduces to 3.14 which is very close to Gaussian behavior. This is due to the fact that larger number of users makes the MAI approaching to the Gaussian behavior by virtue of the central limit theorem.

Figure 5 shows the effect of diversity on the PDF of the MAI-plus-noise. Even though a MIMO-CDMA system will provide higher data rates with an increase in reliability, the system will be severely degraded by such limiting factors as capacity and diversity. The results shown here motivate the fact that any receiver design for such a system will have to take such factors into account.

\subsection{Comparing proposed MAI statistics with the SGA}

In this section, we compare the proposed MAI statistics with the well-known existing approximation of SGA. For this purpose, we use $4 \times 4$ MIMO CDMA systems. Number of users selected are $K=10$ and $K=20$ for the two separate experiments. These results are reported in Figs. 6 and 7 , respectively. For the theoretical result, we plotted 


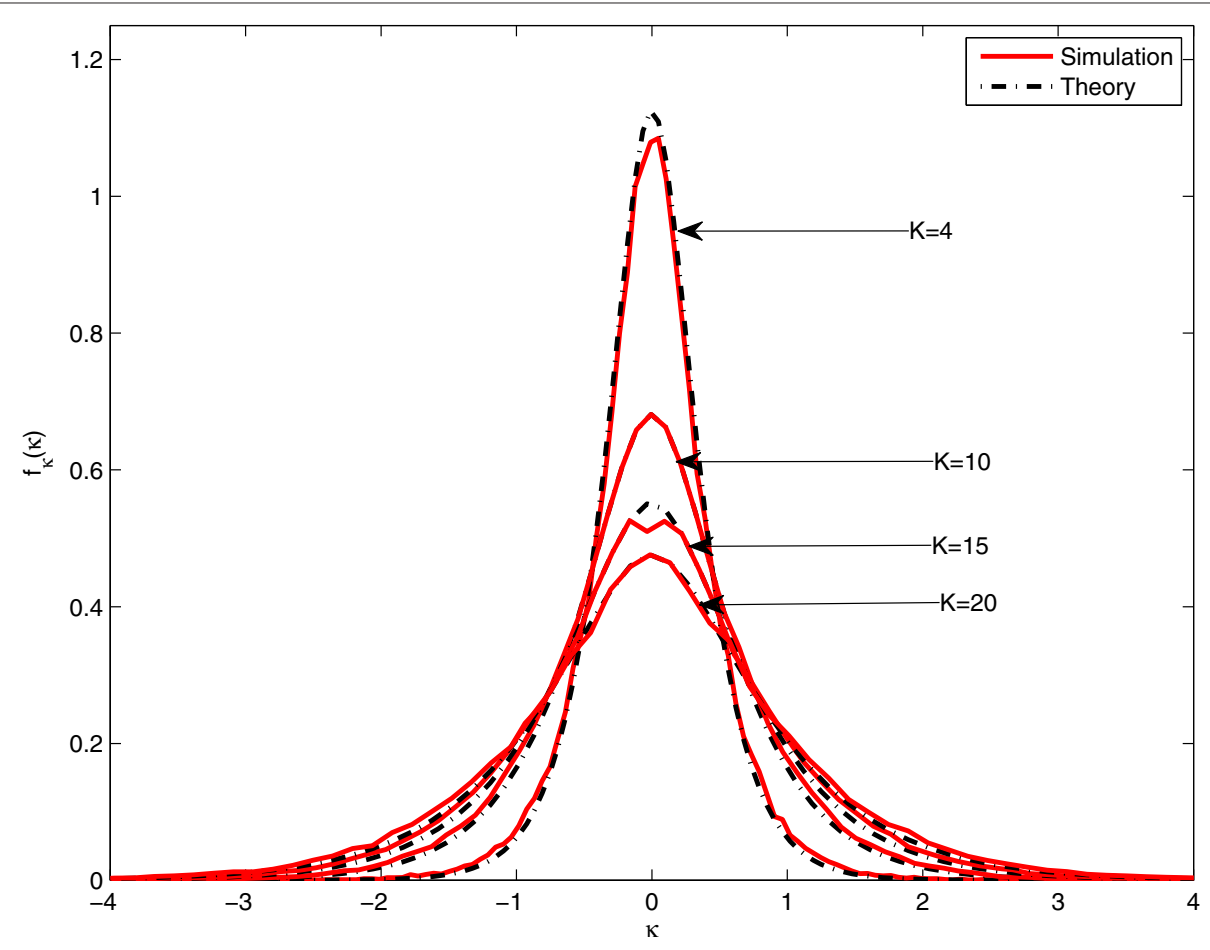

Fig. 4 PDF of MAI-plus-noise under different numbers of users in the system

the PDF of MAI derived in (12). It can be easily depicted from these results that the well-known SGA is failed to correctly model the statistics of the MAI in the MIMOCDMA systems. On the other hand, the proposed analysis provides a good match with the simulation results in both scenarios which shows that the derived theoretical results for the PDF of MAI are valid for both smaller and larger number of users. This also justifies our claim that the MAI cannot be approximated as Gaussian distributed random variable in the MIMO-CDMA systems.

\subsection{Probability of bit error rate}

To validate the theoretical findings, simulations are carried out and results are discussed below. The simulation setup used random signature sequences of length 31 and rectangular chip waveforms. The measurement noise is taken to be additive white Gaussian with an SNR of $20 \mathrm{~dB}$.

Table 2 Experimental kurtosis of MAl-plus-noise under different system's capacity with $N_{C}=31$

\begin{tabular}{llll}
\hline MIMO system & $2 \times 2$ & $3 \times 3$ & $4 \times 4$ \\
\hline$K=4$ & 3.77 & 4.28 & 4.63 \\
$K=10$ & 3.30 & 3.90 & 4.02 \\
$K=15$ & 3.18 & 3.80 & 3.94 \\
$K=20$ & 3.14 & 3.71 & 3.83 \\
\hline
\end{tabular}

Figure 8 shows the performance of CDMA systems in a flat Rayleigh fading environment for a $2 \times 2 \mathrm{MIMO}$ system and a $1 \times 1$ SISO system [16] where the number of system users is set to $K=5$ and $K=15$. It is evident from the result that the proposed analytical findings closely match the experimental ones. It is also evident that the MIMO system performs significantly better by achieving a lower BER as compared to the SISO system, especially for the lower number of users $K=5$. However, an increase in the number of users severely degrades the BER performance due to the resulting increase in MAI. This intuitive fact is well-corroborated by our analysis. The theory-simulation mismatch in the low SNR is mainly due to the relatively high noise power. Moreover, the sum of Rayleigh fading used in the derivation of BER is only an approximation valid for small arguments only. This also contributes somewhat to this mismatch.

Figure 9 shows the BER performance of a MIMOCDMA system versus the number of users in the system. Again, and as expected, we see a close match between the simulation and analytical results. When compared to the SISO system in [16], the MIMO-CDMA system achieves a significantly improved BER performance. It can be seen that the BER performance of the system degrades as the MAI experienced by each user increases. 


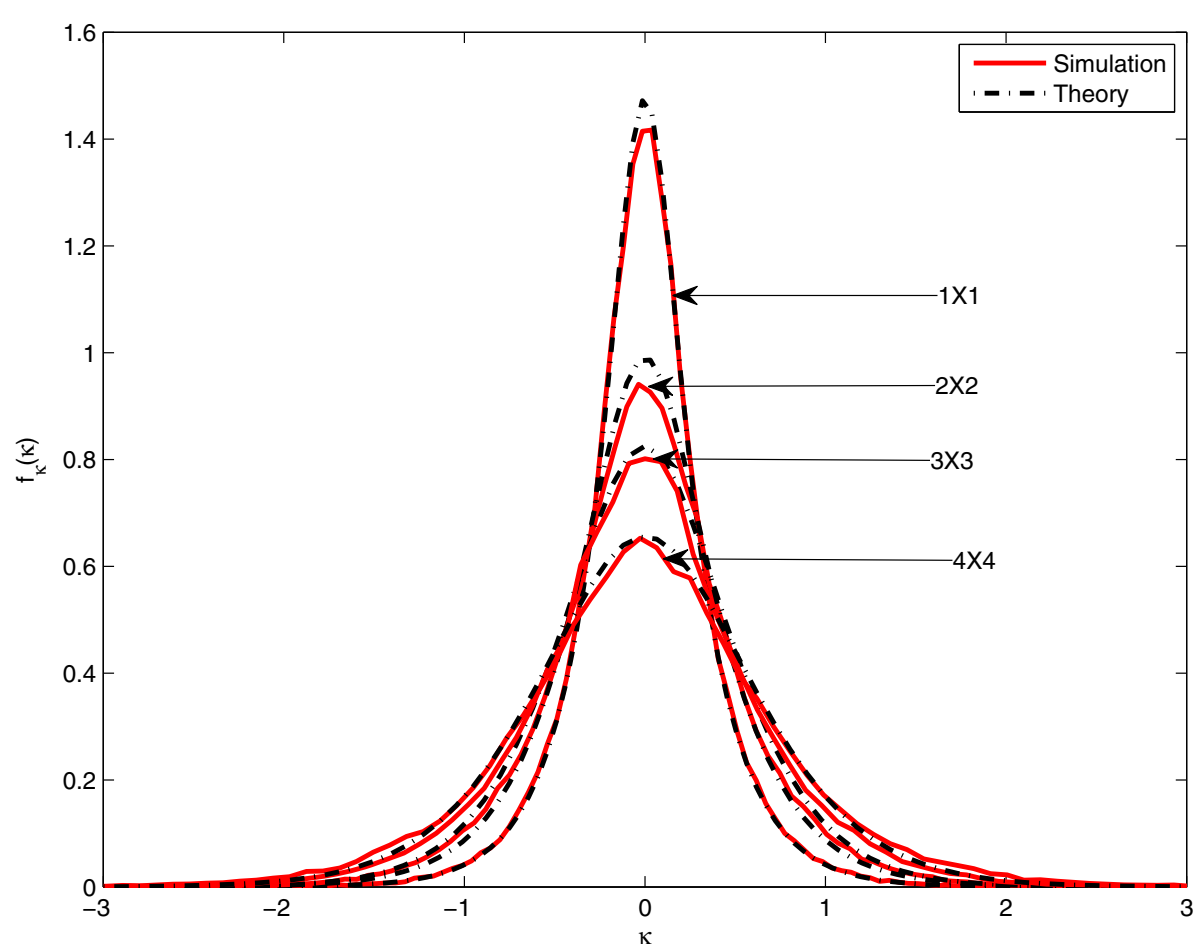

Fig. 5 PDF of MAI-plus-noise for different scenarios of transmit and receive antennas

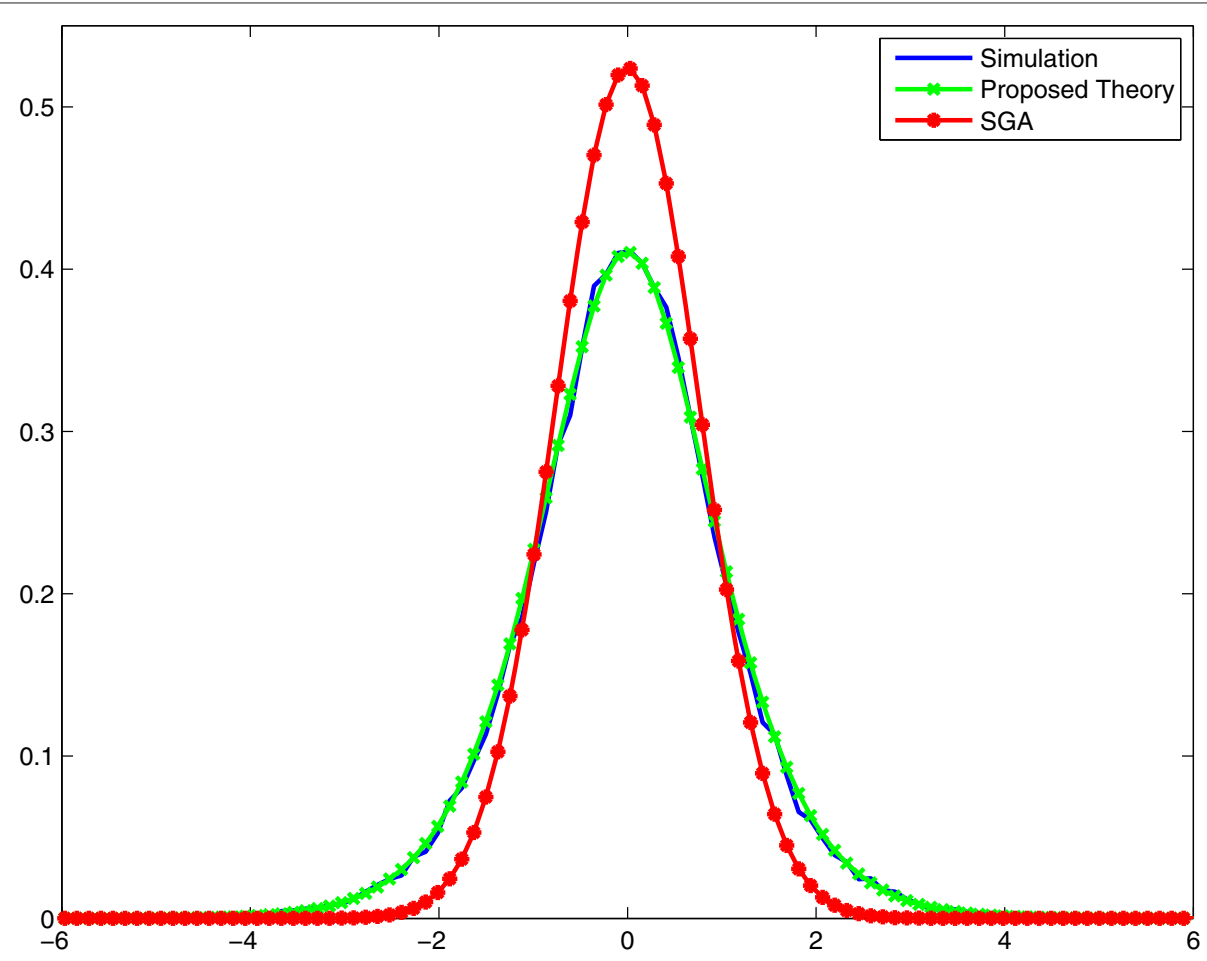

Fig. 6 Comparing the PDF of MAI with SGA in MIMO-CDMA systems for $K=10$ 


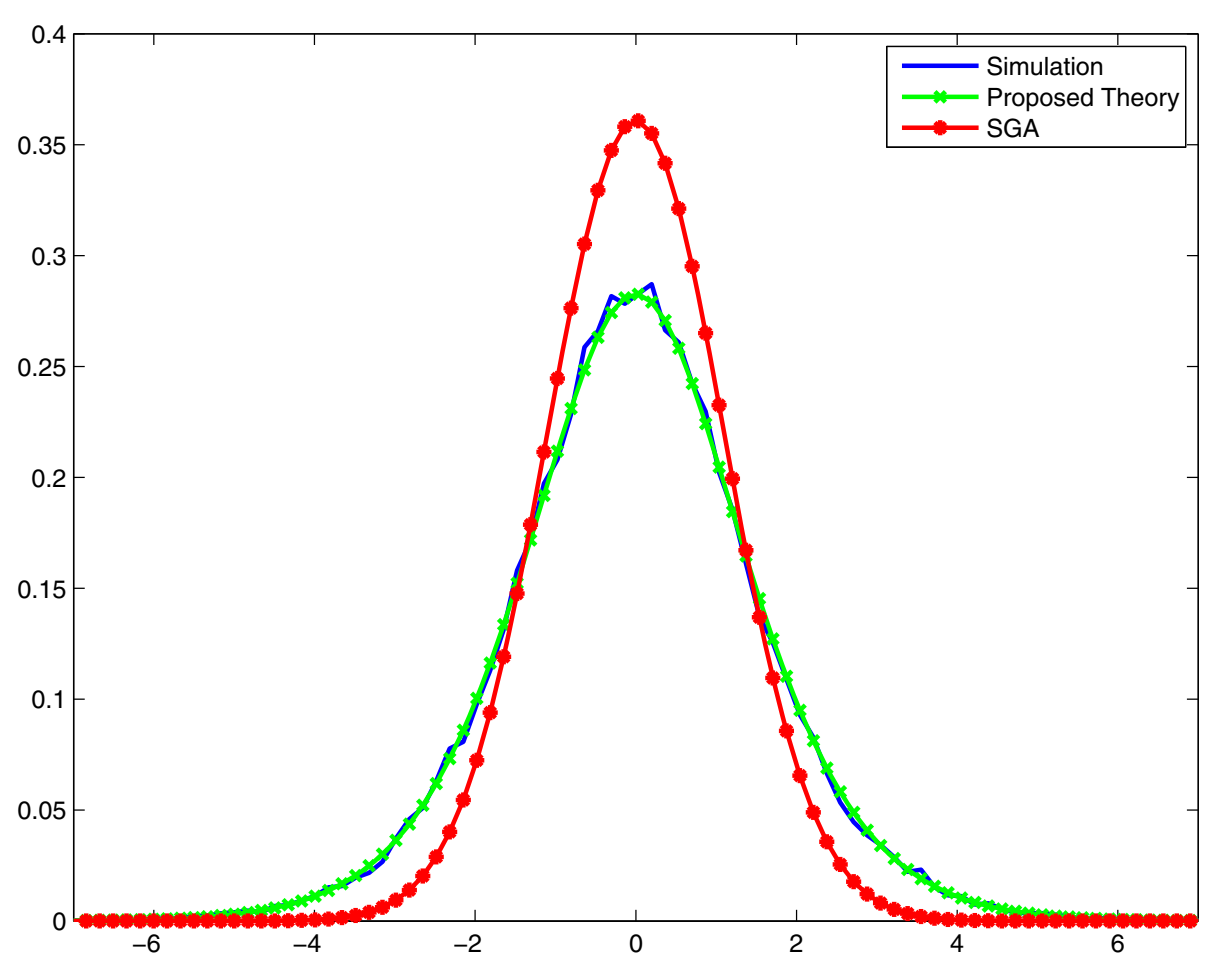

Fig. 7 Comparing the PDF of MAI with SGA in MIMO-CDMA systems for $K=20$

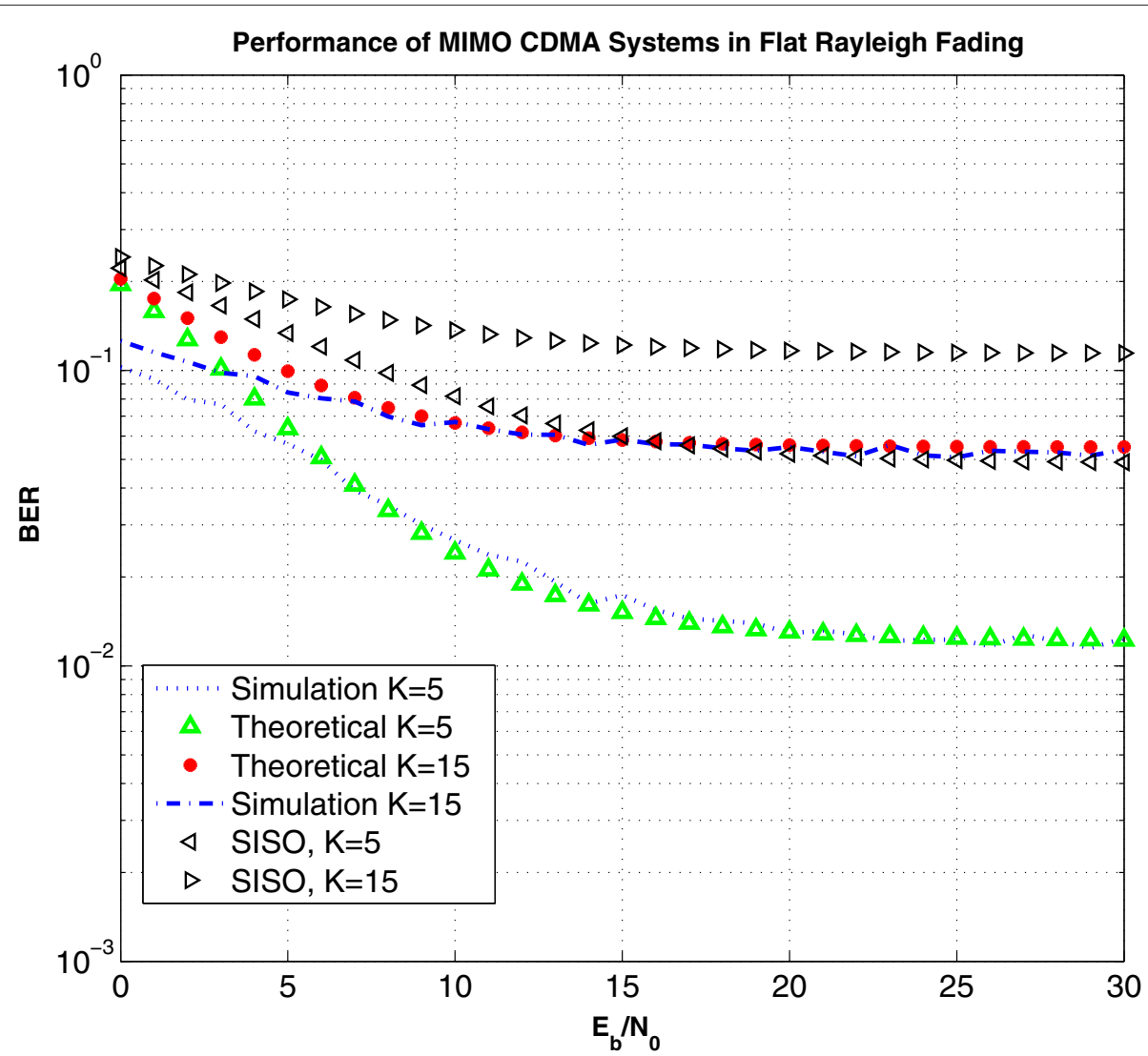

Fig. 8 BER performance of MIMO-CDMA system versus SNR 


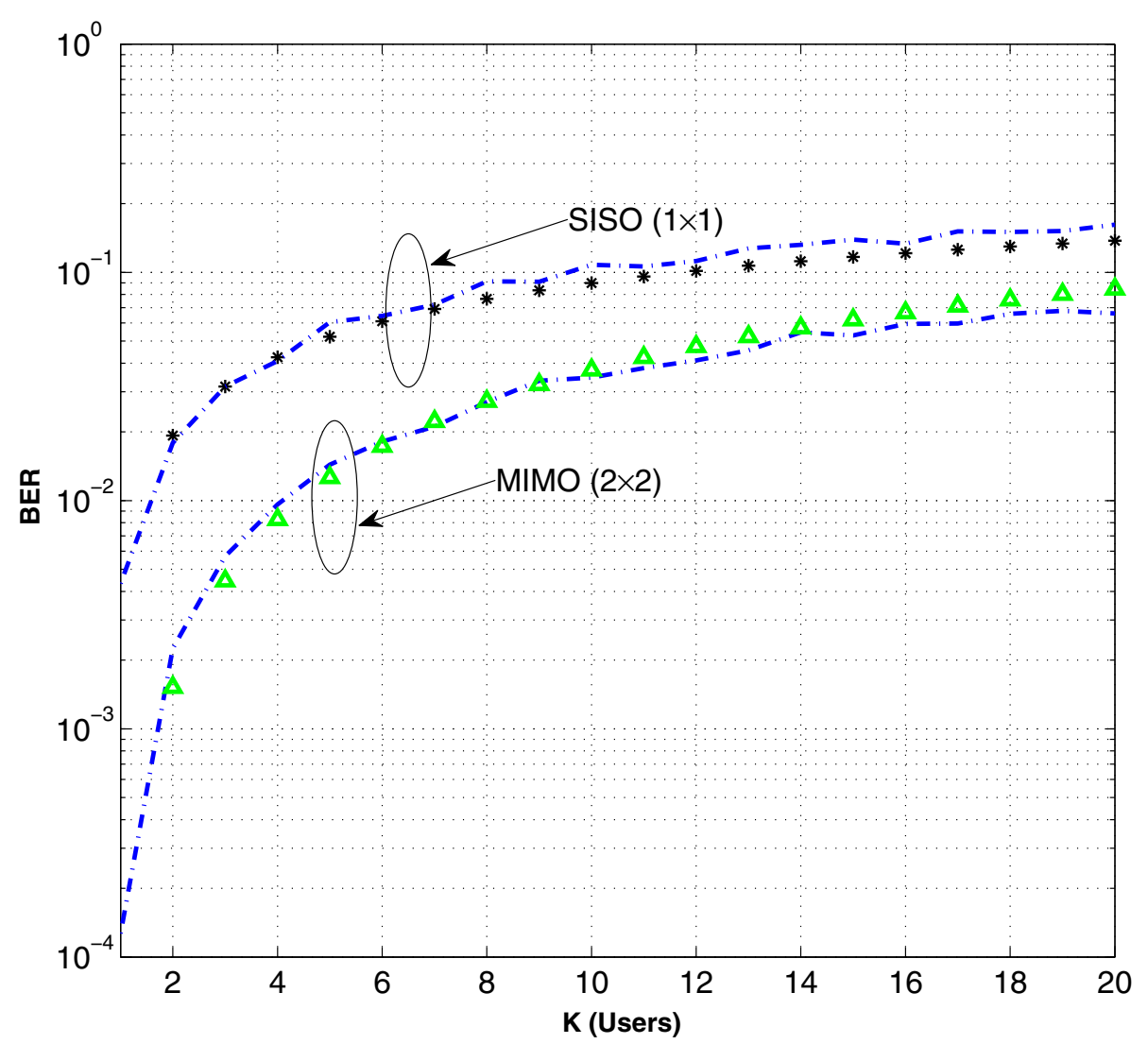

Fig. 9 BER Performance of MIMO-CDMA System versus number of users

\subsection{Channel estimation}

In this simulation, we have used a $2 \times 2$ MIMO system to estimate the channel taps for the flat Rayleigh fading channel based on (37) and (45). The estimator was tested for $K=4, K=6$, and $K=10$ users. Figure 10 shows the mean-square-error performance of the estimator versus the SNR for a flat MIMO Rayleigh channel. As is evident from Fig. 10, the MMSE estimator based on our analysis is able to estimate the channel by minimizing the error for $K=4$ users. As the MAI severely degrades the performance of the system, the estimator is unable to track channel variations for a higher number of users in the system, as is seen for the case of $K=6$ and $K=10$ in the Fig. 10. This is mainly due to the fact that any existing useful information on the channel is simple swamped by the accumulated effect of the contributions to the MAI by the large number of users.

\subsection{Probability of outage}

In this section, we compare the results for the probability of outage obtained via simulation and the proposed theoretical results given in (57). The results are compared for four users (i.e. $K=4$ ). The other system parameters are the same as mentioned in previous experiments. The results are shown in Fig. 11. It can be observed that there is an excellent agreement between the simulation result and the one obtained via the proposed theoretical analysis.

\section{Conclusions}

In this work, a thorough statistical analysis of MAI and MAI-plus-noise in MIMO-CDMA systems has been performed in the presence of Rayleigh fading channel. the major contribution of this work is the statistical characterization of MAI without relying on any Gaussian assumption as is usually reported in the literature. Consequently, the analysis results in new closed-form expressions for the PDF of MAI and MAI-plus-noise. It is found that the derived PDF of MAI is in the form of summation of Laplacian distributed random variables while the PDF of MAI-plus-noise is found to be summation of generalized incomplete gamma functions. These PDFs are found to be a function of key factors such as the number of users, number of antennas, spreading code length, channel variance and noise variance. Moreover, the effect of these parameters on the PDF of MAI has been investigated through simulations whose results have shown a close agreement with the theoretical findings. As applications, we have demonstrated how the derived statistics can 


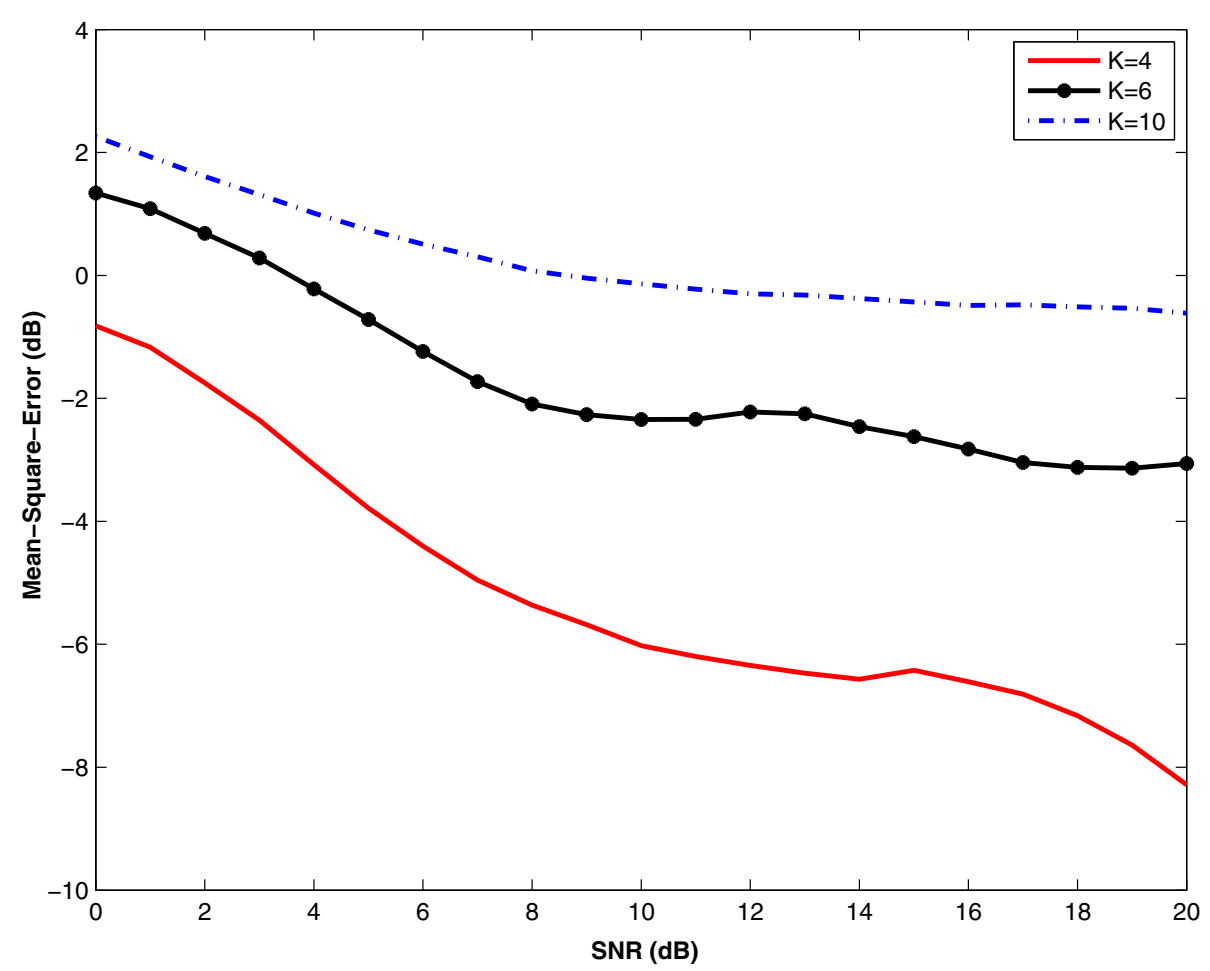

Fig. 10 Mean-square-error performance of the proposed channel estimator

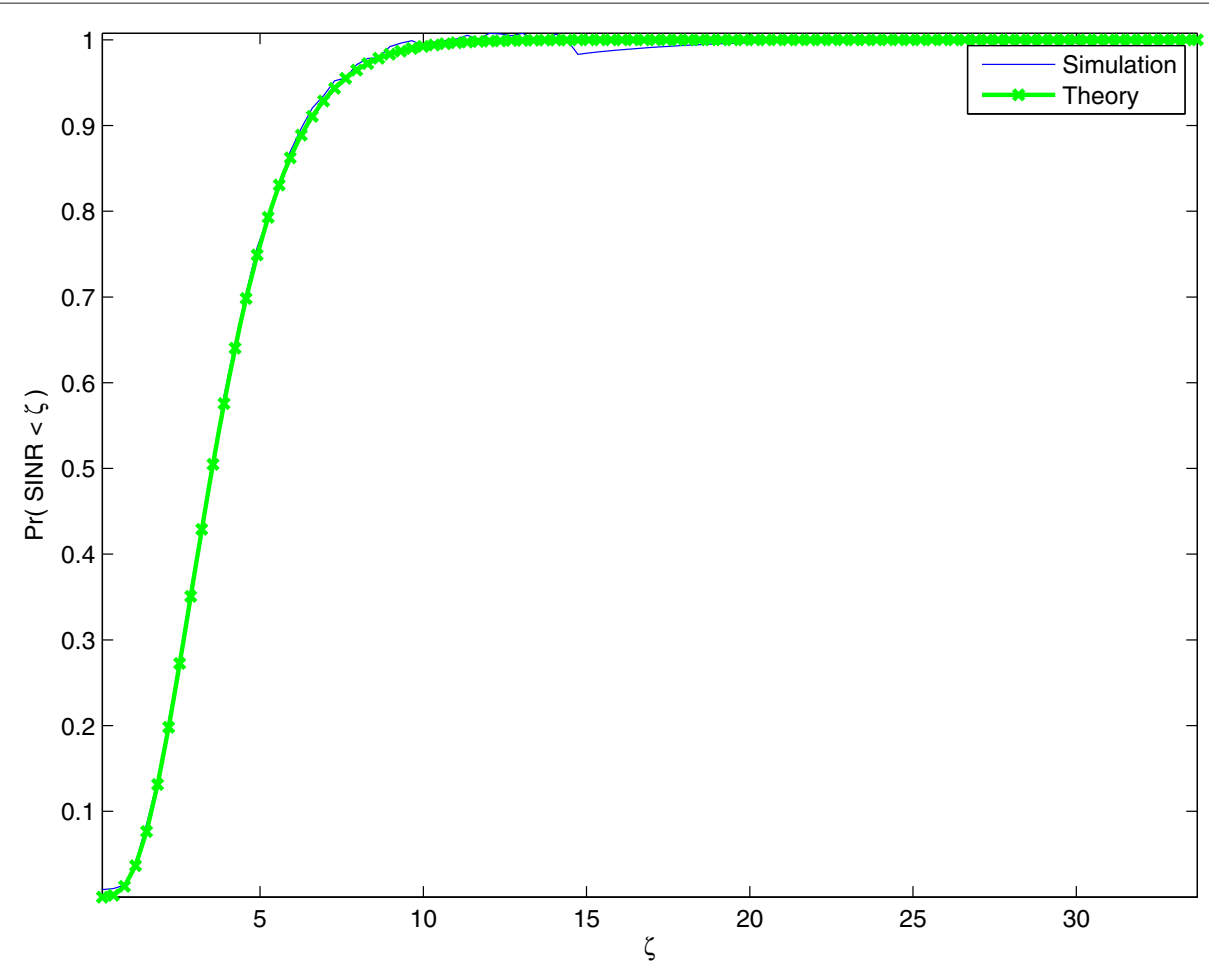

Fig. 11 Probability of outage for Rayleigh fading in MIMO-CDMA systems 
be utilized for designing the optimum coherent receiver, derivation for the expression of probability of bit error rate, derivation of MMSE channel estimator for Rayleigh fading, and derivation for the probability of outage in fading environment. For future work, our analysis can provide a platform for many interesting applications in MIMO-CDMA systems. For example, one can utilize the expression for probability of outage to design a receiver that can optimization the system performance by minimizing the probability of outage. Another interesting application can be the design of optimum algorithm for MIMO antenna selection by joint minimization of receive probability of outage. In addition, our work can extended to frequency selective channels and asynchronous systems. Thus, we believe that our work will open new research directions to solve various challenging problems in MIMO communication systems.

\section{Endnote}

${ }^{1}$ Note that $\sigma_{U_{n}}^{2}$ is just an intermediate variable and it does not represent the true variance of the random variable $U_{n}^{l}$. The actual variance of the $U_{n}^{l}$ is equal to $2 \sigma_{I}^{2} \sigma_{h_{m n}}^{2}$ as $E\left[\left(h_{m n}^{l}\right)^{2}\right]=2 \sigma_{h_{m n}}^{2}$.

\section{Appendix A}

Evaluation of $\frac{1}{2 \pi} \int_{-\infty}^{\infty} \frac{\exp (-i \omega z)}{\prod_{n=1}^{N}\left(\omega^{2} \sigma_{U_{n}}^{2}+1\right)} d \omega$

To evaluate the integral in (11), we consider two different scenarios: (1) $\sigma_{U_{n}}^{2}$ have distinct values for each $n$ and (2) $\sigma_{U_{n}}^{2}$ are equal for all $n$.

Scenario 1: For distinct $\sigma_{U_{n}}^{2}$

For the first scenario, we perform a partial fraction expansion of the product in (10) as follows:

$$
\prod_{n=1}^{N} \frac{1}{\omega^{2} \sigma_{U_{n}}^{2}+1}=\sum_{n=1}^{N} \frac{C_{n}}{\omega^{2} \sigma_{U_{n}}^{2}+1}
$$

where constant $C_{n}$ in the numerator is given by

$$
C_{n}=\frac{\left(\sigma_{U_{n}}^{2}\right)^{N-1}}{\prod_{j=1, j \neq n}^{N}\left(\sigma_{U_{n}}^{2}-\sigma_{U_{j}}^{2}\right)}
$$

Thus, the inverse Fourier transform can be set up as

$$
\begin{aligned}
f_{Z}(z) & =\mathcal{F}^{-1}\left[\Phi_{Z}(\omega)\right] \\
& =\frac{1}{2 \pi} \int_{-\infty}^{\infty} \exp (-i \omega z) \sum_{n=1}^{N} \frac{C_{n}}{\omega^{2} \sigma_{U_{n}}^{2}+1} d \omega \\
& =\sum_{n=1}^{N} \frac{C_{n}}{2 \pi} \int_{-\infty}^{\infty} \frac{\exp (-i \omega z)}{\omega^{2} \sigma_{U_{n}}^{2}+1} d \omega
\end{aligned}
$$

which is a sum of $N$ inverse Fourier transforms. The inner integral can be easily evaluated using the residue theory [24] as

$$
\frac{1}{2 \pi} \int_{-\infty}^{\infty} \frac{e^{-i \omega z}}{\omega^{2} \sigma_{U_{n}}^{2}+1} d \omega=\frac{e^{-\frac{z}{\sigma} U_{n}}}{2 \sigma_{U_{n}}}\left(e^{\frac{2 z}{\sigma U_{n}} \theta(-z)+\theta(z)}\right)
$$

where $\theta(z)$ is the unit step function. The result in (61) can be simplified as

$$
\frac{1}{2 \pi} \int_{-\infty}^{\infty} \frac{e^{-i \omega z}}{\omega^{2} \sigma_{U_{n}}^{2}+1} d \omega=\frac{1}{2} \frac{e^{-\frac{|z|}{\sigma U_{n}}}}{\sigma_{U_{n}}}
$$

Finally, after substituting the result for the above integral in (60), the PDF of the MAI with distinct $\sigma_{U_{n}}^{2}$ is found to be given in (12).

Scenario 2: For identical $\sigma_{U_{n}}^{2}$

For the second scenario of identical $\sigma_{U_{n}}^{2}$ (say $\sigma_{U_{n}}^{2}=\sigma_{U}^{2}$ for all $n$ ), we can set up the integral in (11) as

$$
\frac{1}{2 \pi} \int_{-\infty}^{\infty} \frac{\exp (-i \omega z)}{\left(\omega^{2} \sigma_{U}^{2}+1\right)^{N}} d \omega
$$

which can be solved using the residue theory [24] to obtain

$$
\frac{1}{2 \pi} \int_{-\infty}^{\infty} \frac{\exp (-i \omega z)}{\left(\omega^{2} \sigma_{U}^{2}+1\right)^{N}} d \omega=\frac{1}{\left(2 \sigma_{U}\right)^{N}} \frac{(|z|)^{N-1}}{\Gamma(N)} W_{0, N-\frac{1}{2}}\left(\frac{2|z|}{\sigma_{U}}\right)
$$

where $W_{\lambda, \mu}(z)$ is called Whittaker function and it is defined as [24]

$$
W_{\lambda, \mu}(z)=\frac{z^{\mu+\frac{1}{2}} e^{-z / 2}}{\Gamma\left(\mu-\lambda+\frac{1}{2}\right)} \int_{0}^{\infty} e^{-z t} t^{\mu-\lambda+\frac{1}{2}}(1+t)^{\mu+\lambda+\frac{1}{2}} d t
$$

For the special case when $\lambda=0$, the Whittaker function can be related to the Bessel function for imaginary arguments (denoted as $K_{\mu}(z)$ ) as follows [24]

$$
W_{0, \mu}(z)=\sqrt{\frac{z}{\pi}} K_{\mu}\left(\frac{z}{2}\right)
$$

where, the Bessel function for imaginary arguments is defined as [24]

$K_{\mu}(z)=\frac{1}{2}\left(\frac{z}{2}\right)^{\mu} \int_{0}^{\infty} \frac{e^{-t-z^{2} / 4 t}}{t^{\mu+1}} d t, \quad|\arg (z)|<\frac{\pi}{2}, \quad \operatorname{Re}\left(z^{2}\right)>0$

Thus, by using the above relation, we can simplify the result of integral in (64) to

$$
\frac{1}{2 \pi} \int_{-\infty}^{\infty} \frac{\exp (-i \omega z)}{\left(\omega^{2} \sigma_{U}^{2}+1\right)^{N}} d \omega=\sqrt{\frac{2|z|}{\pi \sigma_{U}}} \frac{1}{\left(2 \sigma_{U}\right)^{N}} \frac{(|z|)^{N-1}}{\Gamma(N)} K_{N-\frac{1}{2}}\left(\frac{|z|}{\pi \sigma_{U}}\right)
$$


Finally, the PDF of the MAI with identical $\sigma_{U_{n}}^{2}$ is obtained after substituting the above solution and it is given in (12).

\section{Derivation of Eq. (32)}

Using (23) and (50) and after some mathematical manipulation, the PDF of $\gamma_{\kappa}$ can be setup as

$$
f_{\gamma_{\kappa}}\left(\gamma_{\kappa}\right)=\frac{\gamma_{\kappa}^{N-1}\left(\frac{N}{\overline{\gamma_{\kappa}}}\right)^{N} \exp \left(-\frac{N \gamma_{\kappa}}{\bar{\gamma}_{\kappa}}\right)}{(N-1) !}
$$

For $N=1$, the PDF of $\gamma_{\kappa}$ reduces to

$$
f_{\gamma_{\kappa}}\left(\gamma_{\kappa}\right)=\frac{1}{\overline{\gamma_{\kappa}}} e^{-\gamma_{\kappa} / \overline{\gamma_{\kappa}}}
$$

which is consistent with [16]. As the channel attenuation is taken to be deterministic then $\gamma_{\kappa}$ is also deterministic. If $\alpha_{i}$ is taken to be random then the above conditional PDF in (26) will have to be averaged over the PDF of $\gamma_{\kappa}$. So the average probability of error in BPSK symbols can be obtained as

$$
\begin{aligned}
P(e)= & \int_{0}^{\infty} P\left(e \mid w_{i, 1}\right) p\left(\gamma_{\kappa}\right) d \gamma_{\kappa} \\
= & \frac{1}{2} \sum_{j=1}^{N} C_{j} \exp \left(\frac{\sigma_{\eta}^{2}}{2 \sigma_{I}^{2} \sigma_{\alpha}^{2}}\right) \int_{0}^{\infty}\left[\int_{\sigma_{\eta}^{2} / 2 \sigma_{I}^{2} \sigma_{\alpha}^{2}}^{\infty} e^{-t} \operatorname{erfc}\left(\sqrt{\gamma_{\kappa}}\right) d t\right] \\
& \times \frac{\gamma_{\kappa}^{N-1}\left(\frac{N}{\overline{\gamma_{\kappa}}}\right)^{N} \exp \left(-\frac{N \gamma_{\kappa}}{\gamma_{\kappa}}\right)}{(N-1) !} d \gamma_{\kappa}
\end{aligned}
$$

Rearranging the above we get

$$
\begin{aligned}
P(e)= & \frac{1}{2} \sum_{j=1}^{N} C_{j} \exp \left(\frac{\sigma_{\eta}^{2}}{2 \sigma_{I}^{2} \sigma_{\alpha}^{2}}\right) \int_{\sigma_{\eta}^{2} / 2 \sigma_{I}^{2} \sigma_{\alpha}^{2}}^{\infty} e^{-t}\left(\frac{N}{\overline{\gamma_{\kappa}}}\right)^{N} \\
& \times\left[\int_{0}^{\infty} \gamma_{\kappa}^{N-1} \exp \left(-\frac{N \gamma_{\kappa}}{\overline{\gamma_{\kappa}}}\right) \operatorname{erfc}\left(\sqrt{\gamma_{\kappa}}\right) d \gamma_{\kappa}\right] d t
\end{aligned}
$$

The inner integral can be evaluated as

$$
\begin{aligned}
& I_{\gamma_{\kappa}}=\int_{0}^{\infty} \gamma_{\kappa}^{N-1} \exp \left(-\frac{N \gamma_{\kappa}}{\overline{\gamma_{\kappa}}}\right) \operatorname{erfc}\left(\sqrt{\gamma_{\kappa}}\right) d \gamma_{\kappa} \\
& I_{\gamma_{\kappa}}=2^{1-2 N} \Gamma(2 N){ }_{2} F_{1}\left(N, N+\frac{1}{2} ; N+1,-\frac{N}{\overline{\gamma_{\kappa}}}\right) / \Gamma(N+1)
\end{aligned}
$$

The above result is again consistent with [16] for $N=1$ which yields $I_{\gamma_{\kappa}}=\overline{\gamma_{\kappa}}\left(1-\sqrt{\frac{\overline{\gamma_{\kappa}}}{1+\overline{\gamma_{\kappa}}}}\right)$. Replacing in the above integral in (72) and rearranging terms, we get

$$
\begin{aligned}
P(e)= & \frac{1}{2} \sum_{j=1}^{N} C_{j} 2^{1-N} \exp \left(\frac{\sigma_{\eta}^{2}}{2 \sigma_{I}^{2} \sigma_{\alpha}^{2}}\right)\left(\frac{\sigma_{I}^{2} \sigma_{\alpha}^{2}}{\psi N^{2} E_{b}}\right)^{N} \frac{\Gamma(2 N)}{\Gamma(N+1)} \\
& \times \int_{\sigma_{\eta}^{2} / 2 \sigma_{I}^{2} \sigma_{\alpha}^{2}}^{\infty} e^{-t} t_{2}^{N} F_{1}\left(N, N+\frac{1}{2} ; N+1,-\frac{2 \sigma_{I}^{2} \sigma_{\alpha}^{2} t}{\psi N^{2} E_{b}}\right) d t
\end{aligned}
$$

\section{Competing interests}

The authors declare that they have no competing interests.

\section{Acknowledgements}

The authors acknowledge the support provided by the Deanship of Scientific Research at KFUPM under Research Grant SB111012.

\section{Author details}

${ }^{1}$ Electrical and Electronics Technology Department, Affiliated Colleges at Hafr Al Batin, King Fahd University of Petroleum and Minerals, P. O. Box 1803, Hafr Al Batin 31991, Saudi Arabia. ${ }^{2}$ Electrical and Computer Engineering, King Abdulaziz University, Jeddah, Saudi Arabia. ${ }^{3}$ Department of Electrical Engineering, King Fahd University of Petroleum and Minerals, Dhahran 31261, Kingdom of Saudi Arabia. ${ }^{4}$ Department of Systems Engineering, King Fahd University of Petroleum and Minerals, Dhahran 31261, Kingdom of Saudi Arabia.

Received: 9 June 2015 Accepted: 17 March 2016

Published online: 04 April 2016

\section{References}

1. M Pursley, D Sarwate, Performance evaluation for phase-coded spread-spectrum multiple-access communication-part ii: code sequence analysis. Commun. IEEE Trans. 25, 800-803 (1977)

2. RK Morrow Jr, JS Lehnert, Bit-to-bit error dependence in slotted ds/ssma packet systems with random signature sequences. Commun. IEEE Trans. 37, 1052-1061 (1989)

3. JM Holtzman, in Communications, 1991. ICC'91, Conference Record. A simple, accurate method to calculate spread spectrum multiple access error probabilities, vol. 3 (IEEE International Conference on, Denver, CO, 1991), pp. $1633-1636$

4. B Long, J Hu, P Zhang, Method to improve gaussian approximation accuracy for calculation of spread-spectrum multiple-access error probabilities. Electron. Lett. 31, 529-531 (1995)

5. J Lehnert, M Pursley, Error probabilities for binary direct-sequence spread-spectrum communications with random signature sequences. Commun. IEEE Trans. 35, 87-98 (1987)

6. JJM Holtzman, A simple, accurate method to calculate spread-spectrum multiple-access error probabilities. Commun. IEEE Trans. COM-40, 461-464 (1992)

7. D Borth, M Pursley, Analysis of direct-sequence spread-spectrum multiple-access communication over rician fading channels. Commun. IEEE Trans. 27(10), 1566-1577 (1979)

8. C Gardner, J Orr, Fading effects on the performance of a spread spectrum multiple access communication system. Commun. IEEE Trans. 27(1), 143-149 (1979)

9. E Geraniotis, M Pursley, Error probability for direct-sequence spread-spectrum multiple-access communications-part ii: approximations. Commun. IEEE Trans. 30(5), 985-995 (1982)

10. E Geraniotis, Direct-sequence spread-spectrum multiple-access communications over nonselective and frequency-selective rician fading channels. Commun. IEEE Trans. 34(8), 756-764 (1986)

11. MO Sunay, PJ McLane, in Global Telecommunications Conference, 1996. GLOBECOM'96.'Communications: The Key to Global Prosperity. Calculating error probabilities for DS-CDMA systems: when not to use the Gaussian approximation, vol. 3 (IEEE, London, 1996), pp. 1744-1749

12. MO Sunay, PJ McLane, Probability of error for diversity combining in DS CDMA systems with synchronization errors. Eur. Trans. Telecommun. 9(5), 449-463 (1998)

13. J Cheng, NC Beaulieu, Accurate DS-CDMA bit-error probability calculation in Rayleigh fading. Wirel. Commun. IEEE Trans. 1(1), 3-15 (2002)

14. J Lehnert, M Pursley, Error probabilities for binary direct-sequence spread-spectrum communications with random signature sequences. Commun. IEEE Trans. 35, 87-98 (1987)

15. W Mee Jang, L Nguyen, P Bidarkar, MAI and ICI of synchronous downlink MC-CDMA with frequency offset. Wirel. Commun. IEEE Trans. 5, 693-703 (2006)

16. M Moinuddin, AUH Sheikh, A Zerguine, M Deriche, A unified approach to ber analysis of synchronous downlink CDMA systems with random signature sequences in fading channels with known channel phase. EURASIP J. Adv. Signal Process. 2008, 1-13 (2008) 
17. JO Mark, MNM Saad, BB Samir, in National Postgraduate Conference (NPC), 2011. Average BER perfomance and spectral efficiency for MIMO orthogonal MC DS-CDMA system over Nakagami-m fading channels, (Kuala Lumpur, 2011), pp. 1-6

18. P Li, RC de Lamare, R Fa, Multiple feedback successive interference cancellation detection for multiuser mimo systems. Wirel. Commun. IEEE Trans. 10(8), 2434-2439 (2011)

19. H Huang, H Viswanathan, GJ Foschini, in Global Telecommunications Conference, 1999. GLOBECOM '99. Achieving high data rates in CDMA systems using blast techniques, vol. 5, (1999), pp. 2316-2320

20. G Kaddoum, M Coulon, D Roviras, P Chargé, Theoretical performance for asynchronous multi-user chaos-based communication systems on fading channels. Signal Process. 90(11), 2923-2933 (2010)

21. G Kaddoum, F Gagnon, Performance analysis of STBC-CSK communication system over slow fading channel. Signal Process. 93(7), 2055-2060 (2013)

22. K Mahmood, SM Asad, M Moinuddin, A Zerguine, S Paul, in Statistical Signal Processing (SSP), 2014. Statistical analysis of multiple access interference in Rayleigh fading environment for MIMO CDMA systems (IEEE Workshop on, Gold Coast, VIC, 2014), pp. 412-415

23. KMahmood, SM Asad, OB Saeed, M Moinuddin, A Zerguine, in Communications, Signal Processing, and their Applications (ICCSPA), 2015. Rayleigh fading channel estimation using MMSE estimator for MIMO-CDMA systems (International Conference on, Sharjah, 2015), pp. 1-4

24. IS Gradshteyn, IM Ryzhik, Table of Integrals, Series, and Products, 7th edn. (Academic Press, London, U.K., 2007)

25. MA Chaudhry, SM Zubair, On A Class Of Incomplete Gamma Functions with Applications. (Chapman \& Hall CRC, 2002)

26. JG Proakis, Digital communications, 4th edn. (Mc Graw-Hill, United States, 2001)

27. C D'Amours, AO Dahmane, Bit error rate performance of a MIMO-CDMA system employing parity-bit-selected spreading in frequency nonselective Rayleigh fading. Int. J. Antennas Propagation. 2011, 1-8 (2011)

28. J Hu, S Member, NC Beaulieu, accurate simple closed-form approximations to Rayleigh sum distributions and densities. 9(2), 109-111 (2005)

29. MN Iqbal, M Moinuddin, in Multitopic Conference (INMIC), 2011 IEEE 14th International. Pilot-aided rayleigh fading channel estimation using MMSE estimator for DS-CDMA system, (Karachi, 2011), pp. 347-350

30. AJ Hayter, Probability and statistics for engineers and scientists, 4th edn. (Cengage Learning, Mason, OH, United States, 2012)

31. TV K'arm'an, M Biot, Mathematical methods in engineering, (McGraw-Hill, United States, 1940)

\section{Submit your manuscript to a SpringerOpen ${ }^{\circ}$ journal and benefit from:}

- Convenient online submission

- Rigorous peer review

- Immediate publication on acceptance

- Open access: articles freely available online

- High visibility within the field

- Retaining the copyright to your article

Submit your next manuscript at $\gg$ springeropen.com 